\title{
ORDERED INVERSE SEMIGROUPS
}

\author{
BY \\ TÔRU SAITÔ
}

\begin{abstract}
In this paper, we consider two questions: one is to characterize the structure of ordered inverse semigroups and the other is to give a condition in order that an inverse semigroup is orderable.

The solution of the first question is carried out in terms of three types of mappings. Two of these consist of mappings of an $\mathscr{R}$-class onto an $\mathscr{R}$-class, while one of these consists of mappings of a principal ideal of the semilattice $E$ constituted by idempotents onto a principal ideal of $E$.

As for the second question, we give a theorem which extends a well-known result about groups that a group $G$ with the identity $e$ is orderable if and only if there exists a subsemigroup $P$ of $G$ such that $P \cup P^{-1}=G, P \cap P^{-1}=\{e\}$ and $x P x^{-1} \leqq P$ for every $x \in G$.
\end{abstract}

Introduction. This paper is in the line of our systematic study of ordered semigroups. The purpose of this paper is to solve two questions about ordered inverse semigroups.

In [5], we characterized some kind of ordered inverse semigroups which we called proper. In the first place, this paper is concerned with

QUESTION 1. How can we characterize the structure of ordered inverse semigroups in general?

In connection with this question, in the second place, this paper is concerned with

QUESTION 2. What is a characteristic property in order that an inverse semigroup is orderable?

In order to solve Question 1, we make use of the following three mappings. Let $S$ be an inverse semigroup and let $E$ be the set of all idempotents of $S$. Firstly, for $e, f \in E$ such that $f \leqq e$ in the semilattice $E$, a mapping $\psi(e, f)$ of $R_{e}$ into $R_{f}$ is defined by

$$
x \psi(e, f)=f x \text { for every } x \in R_{e} .
$$

Secondly, for $x \in S$, a mapping $\varphi(x)$ of the principal ideal $P(e)$ of the semilattice $E$ generated by $e$ onto some principal ideal of $E$ is defined by

$$
f \varphi(x)=x^{-1} f x \text { for every } f \in P(e),
$$

where $e$ is an element of $E$ such that $x \in R_{e}$. Thirdly, for $x \in S$, a mapping $\lambda(x)$ of $R_{f}$ onto $R_{e}$ is defined by

$$
y \lambda(x)=x y \quad \text { for every } y \in R_{f},
$$

Received by the editors November 10, 1969.

AMS subject classifications. Primary 0670, 2092.

Key words and phrases. Inverse semigroup, left ordered inverse semigroup, ordered inverse semigroup, tree semilattice, left orderability of inverse semigroups, orderability of inverse semigroups. 
where $e$ is an element of $E$ such that $x \in R_{e}$ and $f=e \varphi(x)$.

$\$ 2$ carries the purely algebraic character and we characterize inverse semigroups in terms of these three mappings. In $\S 3$, we give a solution of the corresponding question to Question 1 concerning left ordered inverse semigroups. In $\$ 5$, we give a solution of Question 1.

$\S 6$ is devoted to Question 2. In this section, a condition in order that an inverse semigroup is left orderable and a condition in order that an inverse semigroup is orderable are given.

1. Preliminaries. The terminologies and notations of Clifford and Preston [1] are used throughout.

Let $S$ be an inverse semigroup. By [1, Theorem 1.17], the set $E$ of all idempotents of $S$ forms a commutative idempotent subsemigroup of $S$.

In general, let $E$ be a commutative idempotent semigroup. By [1, Theorem 1.12], $E$ is a semilattice with respect to the natural ordering of $E$. We denote the partial order of the semilattice by $\leqq$.

Let $E$ be a semilattice with respect to a partial order $\leqq$. For $e \in E$, the set $\{f \in E ; f \leqq e\}$ is called the principal ideal of $E$ generated by $e$.

A semigroup $S$ with a simple order $\leqq$ is called a left (right) ordered semigroup if it satisfies the condition that

$$
a \leqq b \text { implies } c a \leqq c b(a c \leqq b c) \text { for every } c \in S .
$$

$S$ is called an ordered semigroup if it satisfies the condition that

$$
a \leqq b \text { implies } c a \leqq c b \text { and } a c \leqq b c \text { for every } c \in S .
$$

Let $S$ be a one-sided ordered semigroup. An element $c$ of $S$ is said to lie between two elements $a$ and $b$ of $S$ if either $a \leqq c \leqq b$ or $b \leqq c \leqq a$. An element $a$ of $S$ is called positive (negative) if $a<a^{2}\left(a^{2}<a\right)$ and is called nonnegative (nonpositive) if $a \leqq a^{2}$ $\left(a^{2} \leqq a\right)$.

Here we list some results from our previous paper.

Lemma 1.1 [3, Lemma 2]. Let $a$ and $b$ be elements of an ordered idempotent semigroup $S$. Then both $a b$ and ba lie between $a$ and $b$.

Lemma 1.2 [3, Lemma 4]. Let $S$ be an ordered commutative idempotent semigroup and let $c$ be an element of $S$ which lies between two elements $a$ and $b$ of $S$. Then $a b \leqq c$ in the semilattice $S$.

A semilattice $E$ is called a tree semilattice if the set $\{f \in E ; f \leqq e\}$ is a simply ordered set for every $e \in E$. Let $e$ be an element of a tree semilattice $E$. The set $U(e)=\{f \in E ; e \prec f\}$ is called the upper set of $e$. We define a binary relation $\sim$ in $U(e)$ by

$$
\text { for } f, g \in U(e), f \sim g \text { if and only if } e \prec f g \text {. }
$$


Then, by [3, Lemma 5], $\sim$ is an equivalence relation in $U(e)$. Each $\sim$-equivalence class is called a branch at $e$. The cardinal number of branches at $e$ is called the branch number at $e$.

Lemma 1.3 [3, Theorem 3 and Corollary of Theorem 14]. Let $S$ be an ordered commutative idempotent semigroup. Then the semilattice $S$ is a tree semilattice, in which the branch number at every element is at most two.

2. A characterization of inverse semigroups. In this section, we give two theorems of purely algebraic character which characterize inverse semigroups.

THEOREM 2.1. Let $S$ be an inverse semigroup and let $E$ be the set of all idempotents of $S$. Then $E$ is a commutative idempotent subsemigroup of $S$ and so forms a semilattice. For each $e \in E$, let $R_{e}$ be the $\mathscr{R}$-class of $S$ which contains $e$. Then $S=\bigcup_{e \in E} R_{e}$ and

(1) if $e, f \in E$ and $e \neq f$, then $R_{e} \cap R_{f}=\square$.

For each pair of elements $e, f \in E$ such that $f \leqq e$, we define a mapping $\psi(e, f)$ of $R_{e}$ into $R_{f}$ by

$$
x \psi(e, f)=f x \quad \text { for every } x \in R_{e}
$$

Then

(2) for every $e \in E, \psi(e, e)$ is the identity mapping of $R_{e}$;

(3) if $e, f, g \in E$ and $g \leqq f \leqq e$, then $\psi(e, f) \psi(f, g)=\psi(e, g)$.

Moreover, for each $x \in S$, we define a one-to-one mapping $\varphi(x)$ of the principal ideal $P(e)$ of $E$ generated by $e$ onto a principal ideal of $E$ by

$$
f \varphi(x)=x^{-1} f x \quad \text { for every } f \in P(e),
$$

where $e$ is the element of $E$ such that $x \in R_{e}$. Then

(4) for each pair of elements $f, g$ in the domain of $\varphi(x), f \leqq g$ if and only if $f \varphi(x)$ $\leqq g \varphi(x)$;

(5) if $e \in E, x \in R_{e}, g \leqq f \leqq e$ and $y=x \psi(e, f)$, then $g \varphi(x)=g \varphi(y)$.

Furthermore, for each $x \in S$, we define a one-to-one mapping $\lambda(x)$ of $R_{f}$ onto $R_{e}$ by

$$
y \lambda(x)=x y \text { for every } y \in R_{f},
$$

where $e$ is the element of $E$ such that $x \in R_{e}$ and $f=e \varphi(x)$. Then

(6) if $e \in E, \quad x \in R_{e}, \quad f=e \varphi(x), \quad y \in R_{f}$ and $g \leqq e$, then $(y \lambda(x)) \psi(e, g)=$ $(y \psi(f, g \varphi(x))) \lambda(x \psi(e, g))$;

(7) if $e \in E, x \in R_{e}, f=e \varphi(x), y \in R_{f}$ and $g \leqq e$, then $g \varphi(y \lambda(x))=g \varphi(x) \varphi(y)$;

(8) if $e \in E, x \in R_{e}, f=e \varphi(x), y \in R_{f}, g=f \varphi(y)$ and $z \in R_{g}$, then $z \lambda(y \lambda(x))=$ $(z \lambda(y)) \lambda(x)$

(9) for each $e \in E$, there is one and only one element in $R_{e}$ such that $x \lambda(x)$ is definable and $x \lambda(x)=x$.

Finally we have 
(10) $x y=(y \psi(f,(e \varphi(x)) f)) \lambda\left(x \psi\left(e,((e \varphi(x)) f) \varphi(x)^{-1}\right)\right)$, where $e$ and $f$ are the elements of $E$ such that $x \in R_{e}$ and $y \in R_{f}$.

Proof. Since $S$ is an inverse semigroup, $E$ is a commutative idempotent subsemigroup of $S$ and so forms a semilattice with respect to the natural ordering. Since every $\mathscr{R}$-class of $S$ has one and only one idempotent by [1, Theorem 1.17], we have $S=\bigcup_{e \in E} R_{e}$ and also the condition (1). For each element $x \in S$, we denote by $e(x)$ the uniquely determined idempotent $e$ of $S$ such that $x \mathscr{R} e$. Thus, for $e \in E$, $x \in R_{e}$ is equivalent to $e(x)=e$ and also is equivalent to $e=x x^{-1}$, since $x \mathscr{R} x x^{-1}$ and $x x^{-1} \in E$. Now we suppose that $e, f \in E, f \leqq e$ and $x \in R_{e}$. Then

$$
e(x \psi(e, f))=(f x)(f x)^{-1}=f x x^{-1} f=f e f=f .
$$

Hence $\psi(e, f)$ is really a mapping of $R_{e}$ into $R_{f}$. Since $x \psi(e, e)=e x=x x^{-1} x=x$, we have the condition (2). We suppose that $e, f, g \in E$ and $g \leqq f \leqq e$. Then, for $x \in R_{e}$, we have $x \psi(e, f) \in R_{f}$ and so $x \psi(e, f) \psi(f, g)$ is definable and $x \psi(e, f) \psi(f, g)=g(f x)$ $=(g f) x=g x=x \psi(e, g)$. Hence we have the condition (3). Next we suppose that $x \in R_{e}$ and $f \in P(e)$. Then $f \leqq e=x x^{-1}$ and so

$$
\left(x^{-1} x\right)(f \varphi(x))=\left(x^{-1} x\right)\left(x^{-1} f x\right)=x^{-1} f x=f \varphi(x) .
$$

Hence $f \varphi(x) \leqq x^{-1} x$. Therefore $\varphi(x)$ is a mapping of $P(e)=P\left(x x^{-1}\right)$ into $P\left(x^{-1} x\right)$. Similarly $\varphi\left(x^{-1}\right)$ is a mapping of $P\left(x^{-1} x\right)$ into $P\left(x x^{-1}\right)$. But, for $f \in P\left(x x^{-1}\right)$, $f \varphi(x) \varphi\left(x^{-1}\right)=x\left(x^{-1} f x\right) x^{-1}=\left(x x^{-1}\right) f\left(x x^{-1}\right)=f$. Hence $\varphi(x) \varphi\left(x^{-1}\right)$ is the identity mapping of $P\left(x x^{-1}\right)$. Similarly $\varphi\left(x^{-1}\right) \varphi(x)$ is the identity mapping of $P\left(x^{-1} x\right)$. Hence $\varphi(x)$ is a one-to-one mapping of $P(e)=P\left(x x^{-1}\right)$ onto $P\left(x^{-1} x\right)$ and $\varphi(x)^{-1}$ $=\varphi\left(x^{-1}\right)$. We suppose that $e \in E, x \in R_{e}$ and $f, g \in P(e)$. If $f \leqq g$, then $f \leqq g \leqq x x^{-1}$ and so

$$
(f \varphi(x))(g \varphi(x))=\left(x^{-1} f x\right)\left(x^{-1} g x\right)=x^{-1} f x x^{-1} g x=x^{-1} f g x=x^{-1} f x=f \varphi(x) .
$$

Hence $f \varphi(x) \leqq g \varphi(x)$. Conversely, if $f \varphi(x) \leqq g \varphi(x)$, then $f=f \varphi(x) \varphi\left(x^{-1}\right) \leqq g \varphi(x) \varphi\left(x^{-1}\right)$ $=g$. Thus we have the condition (4). We suppose that $e \in E, x \in R_{e}$ and $g \leqq f \leqq e$. Then $y=x \psi(e, f)$ is definable and $e(y)=f$. Hence $g \varphi(x)$ and $g \varphi(y)$ are definable and $g \varphi(y)=y^{-1} g y=(f x)^{-1} g(f x)=x^{-1} f g f x=x^{-1} g x=g \varphi(x)$. Thus we have the condition (5). Next we suppose that $e \in E, x \in R_{e}, f=e \varphi(x)$ and $y \in R_{f}$. Then $f=x^{-1} e x$ $=x^{-1}\left(x x^{-1}\right) x=x^{-1} x$, and so

$$
\begin{aligned}
e(y \lambda(x)) & =e(x y)=(x y)(x y)^{-1}=x y y^{-1} x^{-1}=x f x^{-1} \\
& =x\left(x^{-1} x\right) x^{-1}=x x^{-1}=e .
\end{aligned}
$$

Hence $\lambda(x)$ is a mapping of $R_{f}$ into $R_{e}$. Since $x^{-1} x=f$, we have $x^{-1} \in R_{f}$ and also $f \varphi\left(x^{-1}\right)=x f x^{-1}=x x^{-1} x x^{-1}=x x^{-1}=e$. Hence, in a similar way, $\lambda\left(x^{-1}\right)$ is a mapping of $R_{e}$ into $R_{f}$. Moreover, for $y \in R_{f}$,

$$
y \lambda(x) \lambda\left(x^{-1}\right)=x^{-1}(x y)=\left(x^{-1} x\right) y=f y=y y^{-1} y=y .
$$


Hence $\lambda(x) \lambda\left(x^{-1}\right)$ is the identity mapping of $R_{f}$. Similarly $\lambda\left(x^{-1}\right) \lambda(x)$ is the identity mapping of $R_{e}$. Hence $\lambda(x)$ is a one-to-one mapping of $R_{f}$ onto $R_{e}$. We suppose that $e \in E, x \in R_{e}, f=e \varphi(x), y \in R_{f}$ and $g \leqq e$. Then, since $y \lambda(x) \in R_{e},(y \lambda(x)) \psi(e, g)$ is definable and $(y \lambda(x)) \psi(e, g)=g x y$. Since $g \varphi(x) \leqq e \varphi(x)=f$ and $y \in R_{f}, y \psi(f, g \varphi(x))$ is definable and, since $g \leqq e, x \psi(e, g)$ is definable. Moreover

$$
\begin{gathered}
e(x \psi(e, g)) \varphi(x \psi(e, g))=e(g x) \varphi(g x)=(g x)^{-1}(g x)=x^{-1} g x, \\
y \psi(f, g \varphi(x)) \in R_{g \varphi(x)}=R_{x}^{-1} g x .
\end{gathered}
$$

Hence $(y \psi(f, g \varphi(x))) \lambda(x \psi(e, g))$ is definable and

$$
\begin{aligned}
(y \psi(f, g \varphi(x))) \lambda(x \psi(e, g)) & =(x \psi(e, g))(y \psi(f, g \varphi(x))) \\
& =(g x)\left(x^{-1} g x y\right)=g e g x y=g x y .
\end{aligned}
$$

Thus we have the condition (6). We suppose that $e \in E, x \in R_{e}, f=e \varphi(x), y \in R_{f}$ and $g \leqq e$. Then, since $g \leqq e=e(y \lambda(x)), g \varphi(y \lambda(x))$ is definable. Also, since $g \leqq e=e(x)$ and $g \varphi(x) \leqq e \varphi(x)=f=e(y), g \varphi(x) \varphi(y)$ is definable. Moreover

$$
g \varphi(y \lambda(x))=g \varphi(x y)=(x y)^{-1} g(x y)=y^{-1} x^{-1} g x y=g \varphi(x) \varphi(y) .
$$

Thus we have the condition (7). We suppose that $e \in E, x \in R_{e}, f=e \varphi(x), y \in R_{f}$, $g=f \varphi(y)$ and $z \in R_{g}$. Then $y \lambda(x)$ and $z \lambda(y)$ are definable and

$$
\begin{aligned}
e(y \lambda(x)) \varphi(y \lambda(x)) & =e(x y) \varphi(x y)=(x y)^{-1}(x y)=y^{-1} x^{-1} x y \\
& =y^{-1} f y=f \varphi(y)=g=e(z), \\
e(z \lambda(y)) & =e(y)=f=e(x) \varphi(x) .
\end{aligned}
$$

Hence $z \lambda(y \lambda(x))$ and $(z \lambda(y)) \lambda(x)$ are definable and

$$
z \lambda(y \lambda(x))=z \lambda(x y)=(x y) z=x(y z)=x(z \lambda(y))=(z \lambda(y)) \lambda(x) .
$$

Thus we have the condition (8). Let $e \in E$. Then $e(e) \varphi(e)=e^{-1} e e=e=e(e)$. Hence $e \lambda(e)$ is definable and $e \lambda(e)=e e=e$. Conversely let $x$ be an element of $R_{e}$ such that $x \lambda(x)$ is definable and $x \lambda(x)=x$. Then $x^{2}=x$ and so $x=e$. Thus we have the condition (9). Finally we suppose that $e, f \in E, x \in R_{e}$ and $y \in R_{f}$. Then

$$
\begin{aligned}
(e \varphi(x)) f \leqq f & =e(y), \\
((e \varphi(x)) f) \varphi(x)^{-1} & =((e \varphi(x)) f) \varphi\left(x^{-1}\right) \leqq e \varphi(x) \varphi\left(x^{-1}\right) \\
& =e \varphi(x) \varphi(x)^{-1}=e=e(x) .
\end{aligned}
$$

Hence $y \psi(f,(e \varphi(x)) f)$ and $x \psi\left(e,((e \varphi(x)) f) \varphi(x)^{-1}\right)$ are definable. Now

$$
\begin{aligned}
x \psi\left(e,((e \varphi(x)) f) \varphi(x)^{-1}\right) & =\left(((e \varphi(x)) f) \varphi\left(x^{-1}\right)\right) x \\
& =\left(x\left(x^{-1} x y y^{-1}\right) x^{-1}\right) x=x y y^{-1} \\
y \psi(f,(e \varphi(x)) f) & =((e \varphi(x)) f) y=\left(x^{-1} x y y^{-1}\right) y=x^{-1} x y
\end{aligned}
$$


and so

$$
\begin{aligned}
e\left(x \psi\left(e,((e \varphi(x)) f) \varphi(x)^{-1}\right)\right) \varphi\left(x \psi\left(e,((e \varphi(x)) f) \varphi(x)^{-1}\right)\right) \\
=\left(x y y^{-1}\right)^{-1}\left(x y y^{-1}\right)=y y^{-1} x^{-1} x y y^{-1}=x^{-1} x y y^{-1} \\
=x^{-1} x y y^{-1} x^{-1} x=\left(x^{-1} x y\right)\left(x^{-1} x y\right)^{-1}=e(y \psi(f,(e \varphi(x)) f)) .
\end{aligned}
$$

Hence $(y \psi(f,(e \varphi(x)) f)) \lambda\left(x \psi\left(e,((e \varphi(x)) f) \varphi(x)^{-1}\right)\right)$ is definable and

$$
(y \psi(f,(e \varphi(x)) f)) \lambda\left(x \psi\left(e,((e \varphi(x)) f) \varphi(x)^{-1}\right)\right)=\left(x y y^{-1}\right)\left(x^{-1} x y\right)=x y .
$$

Thus we have the condition (10). This completes the proof of Theorem 2.1.

Conversely we have

THEOREM 2.2. Let $E$ be a commutative idempotent semigroup. Suppose that, for each $e \in E$, there corresponds a nonempty set $R_{e}$, which satisfies the condition (1) in Theorem 2.1. We put $S=\bigcup_{e \in E} R_{e}$. Suppose that, for every pair of elements $e, f \in E$ such that $f \leqq e$, a mapping $\psi(e, f)$ of $R_{e}$ into $R_{f}$, and, for every $x \in S$, a one-to-one mapping $\varphi(x)$ of the principal ideal $P(e)$ of $E$ onto a principal ideal of $E$, where $e$ is the element of $E$ such that $x \in R_{e}$, and moreover, for every $x \in S$, a one-to-one mapping $\lambda(x)$ of $R_{f}$ onto $R_{e}$, where $e$ is the element of $E$ such that $x \in R_{e}$ and $f=e \varphi(x)$, are given. Suppose that these mappings satisfy the conditions (2)-(9) in Theorem 2.1. $W e$ define the product in $S$ by (10) in Theorem 2.1. Then $S$ is an inverse semigroup.

Moreover, if $y \lambda(x)$ is definable, then $y \lambda(x)=x y$. Also there exists a semigroup and semilattice isomorphism of $E$ onto the commutative idempotent subsemigroup $E^{*}$ of $S$ constituted by all idempotents of $S$ and when, for each $g \in E$, we denote by $g^{*}$ the element of $E^{*}$ corresponding to $g$ by the isomorphism, $R_{e}$ is the $\mathscr{R}$-class of $S$ which contains the element $e^{*}, x \psi(e, f)=f^{*} x$ if $x \psi(e, f)$ is definable, and $(e \varphi(x))^{*}=x^{-1} e^{*} x$ if $e \varphi(x)$ is definable.

Proof. By (1) and the fact that $S=\bigcup_{e \in E} R_{e}$, for each $x \in S$ there exists one and only one $e \in E$ such that $x \in R_{e}$, which we denote by $e(x)$. We divide the proof into several steps.

$1^{\circ}$. If $e \in E, x \in R_{e}, g \leqq f \leqq e$ and $y=x \psi(e, f)$, then $g \varphi(x)$ and $g \varphi(y)$ are definable.

In fact, $g \leqq e=e(x)$ and $g \leqq f=e(y)$, and so $g \varphi(x)$ and $g \varphi(y)$ are definable.

$2^{\circ}$. If $e \in E, x \in R_{e}, f=e \varphi(x), y \in R_{f}$ and $g \leqq e$, then $(y \lambda(x)) \psi(e, g)$ and $(y \psi(f, g \varphi(x))) \lambda(x \psi(e, g))$ are definable.

In fact, since $y \lambda(x) \in R_{e},(y \lambda(x)) \psi(e, g)$ is definable. We have $e(x \psi(e, g))=g \leqq e$ $=e(x)$ and so, by $1^{\circ}$ and (5), $e(x \psi(e, g)) \varphi(x \psi(e, g))=g \varphi(x \psi(e, g))$ is definable and

$$
e(x \psi(e, g)) \varphi(x \psi(e, g))=g \varphi(x) \text {. }
$$

On the other hand, $g \varphi(x) \leqq e \varphi(x)=f=e(y)$ by (4). Hence $y \psi(f, g \varphi(x))$ is definable and

$$
e(y \psi(f, g \varphi(x)))=g \varphi(x)=e(x \psi(e, g)) \varphi(x \psi(e, g)) .
$$

Therefore $(y \psi(f, g \varphi(x))) \lambda(x \psi(e, g))$ is definable. 
$3^{\circ}$. If $e \in E, x \in R_{e}, f=e \varphi(x), y \in R_{f}$ and $g \leqq e$, then $g \varphi(y \lambda(x))$ and $g \varphi(x) \varphi(y)$ are definable.

In fact, $g \leqq e=e(y \lambda(x))$ and so $g \varphi(y \lambda(x))$ is definable. Also, by (4), $g \varphi(x) \leqq e \varphi(x)$ $=f=e(y)$ and so $g \varphi(x) \varphi(y)$ is definable.

$4^{\circ}$. If $e \in E, x \in R_{e}, f=e \varphi(x), y \in R_{f}, g=f \varphi(y)$ and $z \in R_{g}$, then $z \lambda(y \lambda(x))$ and $(z \lambda(y)) \lambda(x)$ are definable.

In fact, by $3^{\circ}$ and (7), both $e(y \lambda(x)) \varphi(y \lambda(x))=e \varphi(y \lambda(x))$ and $e \varphi(x) \varphi(y)$ are definable and

$$
e(y \lambda(x)) \varphi(y \lambda(x))=e \varphi(x) \varphi(y)=f \varphi(y)=g=e(z) .
$$

Hence $z \lambda(y \lambda(x))$ is definable. Also $e(x) \varphi(x)=e \varphi(x)=f=e(z \lambda(y))$ and so $(z \lambda(y)) \lambda(x)$ is definable.

$5^{\circ}$. If $e, f \in E, x \in R_{e}$ and $y \in R_{f}$, then $(y \psi(f,(e \varphi(x)) f)) \lambda\left(x \psi\left(e,((e \varphi(x)) f) \varphi(x)^{-1}\right)\right)$ is definable.

In fact, $(e \varphi(x)) f \leqq f=e(y)$ and so $y \psi(f,(e \varphi(x)) f)$ is definable. By (4),

$$
((e \varphi(x)) f) \varphi(x)^{-1} \leqq e \varphi(x) \varphi(x)^{-1}=e=e(x)
$$

and so $x \psi\left(e,((e \varphi(x)) f) \varphi(x)^{-1}\right)$ is definable. Moreover, by $1^{\circ}$ and (5),

$$
\begin{aligned}
e\left(x \psi\left(e,((e \varphi(x)) f) \varphi(x)^{-1}\right)\right) \varphi\left(x \psi\left(e,((e \varphi(x)) f) \varphi(x)^{-1}\right)\right) \\
=((e \varphi(x)) f) \varphi(x)^{-1} \varphi\left(x \psi\left(e,((e \varphi(x)) f) \varphi(x)^{-1}\right)\right) \\
=((e \varphi(x)) f) \varphi(x)^{-1} \varphi(x)=(e \varphi(x)) f=e(y \psi(f,(e \varphi(x)) f)) .
\end{aligned}
$$

Hence $(y \psi(f,(e \varphi(x)) f)) \lambda\left(x \psi\left(e,((e \varphi(x)) f) \varphi(x)^{-1}\right)\right)$ is definable.

$6^{\circ}$. If $e, f, g \in E, x \in R_{e}, y \in R_{f}$ and $z \in R_{g}$, then, putting

$$
\begin{aligned}
& x_{1}=x \psi\left(e,((((e \varphi(x)) f) \varphi(y)) g) \varphi(y)^{-1} \varphi(x)^{-1}\right), \\
& y_{1}=y \psi\left(f,((((e \varphi(x)) f) \varphi(y)) g) \varphi(y)^{-1}\right), \\
& z_{1}=z \psi(g,(((e \varphi(x)) f) \varphi(y)) g)
\end{aligned}
$$

$z_{1} \lambda\left(y_{1} \lambda\left(x_{1}\right)\right)$ is definable and $(x y) z=z_{1} \lambda\left(y_{1} \lambda\left(x_{1}\right)\right)$.

In fact, by $5^{\circ}$ and (10),

$$
x y=(y \psi(f,(e \varphi(x)) f)) \lambda\left(x \psi\left(e,((e \varphi(x)) f) \varphi(x)^{-1}\right)\right)
$$

and the right-hand side is definable. Hence

$$
e(x y)=e\left(x \psi\left(e,((e \varphi(x)) f) \varphi(x)^{-1}\right)\right)=((e \varphi(x)) f) \varphi(x)^{-1}
$$

and, by $3^{\circ}$ and (7),

$$
\begin{aligned}
e(x y) \varphi(x y) & =((e \varphi(x)) f) \varphi(x)^{-1} \varphi\left((y \psi(f,(e \varphi(x)) f)) \lambda\left(x \psi\left(e,((e \varphi(x)) f) \varphi(x)^{-1}\right)\right)\right) \\
& =((e \varphi(x)) f) \varphi(x)^{-1} \varphi\left(x \psi\left(e,((e \varphi(x)) f) \varphi(x)^{-1}\right)\right) \varphi(y \psi(f,(e \varphi(x)) f))
\end{aligned}
$$


But, in the proof of $5^{\circ}$, it was shown that

$$
((e \varphi(x)) f) \varphi(x)^{-1} \varphi\left(x \psi\left(e,((e \varphi(x)) f) \varphi(x)^{-1}\right)\right)=(e \varphi(x)) f .
$$

Moreover, since $(e \varphi(x)) f \leqq f=e(y)$, we have, by $1^{\circ}$ and (5),

$$
((e \varphi(x)) f) \varphi(y \psi(f,(e \varphi(x)) f))=((e \varphi(x)) f) \varphi(y) .
$$

Hence we obtain

$$
e(x y) \varphi(x y)=((e \varphi(x)) f) \varphi(y) .
$$

Therefore, by $5^{\circ}$ and (10),

$$
(x y) z=z_{1} \lambda\left(y^{\prime} \lambda\left(x^{\prime}\right) \psi\left(((e \varphi(x)) f) \varphi(x)^{-1},((((e \varphi(x)) f) \varphi(y)) g) \varphi\left(y^{\prime} \lambda\left(x^{\prime}\right)\right)^{-1}\right)\right)
$$

and the right-hand side is definable, where

$$
x^{\prime}=x \psi\left(e,((e \varphi(x)) f) \varphi(x)^{-1}\right), \quad y^{\prime}=y \psi(f,(e \varphi(x)) f) .
$$

Now $(((e \varphi(x)) f) \varphi(y)) g \leqq((e \varphi(x)) f) \varphi(y)$. Hence $((((e \varphi(x)) f) \varphi(y)) g) \varphi(y)^{-1}$ is definable and, by (4),

$$
((((e \varphi(x)) f) \varphi(y)) g) \varphi(y)^{-1} \leqq(e \varphi(x)) f \leqq e \varphi(x) .
$$

Therefore $((((e \varphi(x)) f) \varphi(y)) g) \varphi(y)^{-1} \varphi(x)^{-1}$ is definable and, again by (4),

$$
((((e \varphi(x)) f) \varphi(y)) g) \varphi(y)^{-1} \varphi(x)^{-1} \leqq((e \varphi(x)) f) \varphi(x)^{-1}=e\left(x^{\prime}\right) \leqq e=e(x) .
$$

Hence, by $3^{\circ}$ and (7),

$$
\begin{aligned}
\left(((((e \varphi(x)) f) \varphi(y)) g) \varphi(y)^{-1} \varphi(x)^{-1}\right) \varphi\left(y^{\prime} \lambda\left(x^{\prime}\right)\right) \\
=\left(((((e \varphi(x)) f) \varphi(y)) g) \varphi(y)^{-1} \varphi(x)^{-1}\right) \varphi\left(x^{\prime}\right) \varphi\left(y^{\prime}\right)
\end{aligned}
$$

and both sides are definable. Also, by (5),

$$
\begin{aligned}
\left(((((e \varphi(x)) f) \varphi(y)) g) \varphi(y)^{-1} \varphi(x)^{-1}\right) \varphi\left(x^{\prime}\right) \\
=\left(((((e \varphi(x)) f) \varphi(y)) g) \varphi(y)^{-1} \varphi(x)^{-1}\right) \varphi\left(x \psi\left(e,((e \varphi(x)) f) \varphi(x)^{-1}\right)\right) \\
=((((e \varphi(x)) f) \varphi(y)) g) \varphi(y)^{-1} \varphi(x)^{-1} \varphi(x) \\
\quad=((((e \varphi(x)) f) \varphi(y)) g) \varphi(y)^{-1}
\end{aligned}
$$

Since $((((e \varphi(x)) f) \varphi(y)) g) \varphi(y)^{-1} \leqq(e \varphi(x)) f \leqq f$, we have, again by (5),

$$
\begin{aligned}
\left(((((e \varphi(x)) f) \varphi(y)) g) \varphi(y)^{-1}\right) \varphi\left(y^{\prime}\right) \\
=\left(((((e \varphi(x)) f) \varphi(y)) g) \varphi(y)^{-1}\right) \varphi(y \psi(f,(e \varphi(x)) f)) \\
=((((e \varphi(x)) f) \varphi(y)) g) \varphi(y)^{-1} \varphi(y)=(((e \varphi(x)) f) \varphi(y)) g .
\end{aligned}
$$

Hence

$$
\left(((((e \varphi(x)) f) \varphi(y)) g) \varphi(y)^{-1} \varphi(x)^{-1}\right) \varphi\left(y^{\prime} \lambda\left(x^{\prime}\right)\right)=(((e \varphi(x)) f) \varphi(y)) g
$$

and so

$$
((((e \varphi(x)) f) \varphi(y)) g) \varphi\left(y^{\prime} \lambda\left(x^{\prime}\right)\right)^{-1}=((((e \varphi(x)) f) \varphi(y)) g) \varphi(y)^{-1} \varphi(x)^{-1}
$$


Therefore, by $2^{\circ}$ and (6),

$$
\begin{aligned}
& y^{\prime} \lambda\left(x^{\prime}\right) \psi\left(((e \varphi(x)) f) \varphi(x)^{-1},((((e \varphi(x)) f) \varphi(y)) g) \varphi\left(y^{\prime} \lambda\left(x^{\prime}\right)\right)^{-1}\right) \\
&= y^{\prime} \lambda\left(x^{\prime}\right) \psi\left(((e \varphi(x)) f) \varphi(x)^{-1},((((e \varphi(x)) f) \varphi(y)) g) \varphi(y)^{-1} \varphi(x)^{-1}\right) \\
&=\left(y^{\prime} \psi\left((e \varphi(x)) f,((((e \varphi(x)) f) \varphi(y)) g) \varphi(y)^{-1} \varphi(x)^{-1} \varphi\left(x^{\prime}\right)\right)\right) \\
& \cdot \lambda\left(x^{\prime} \psi\left(((e \varphi(x)) f) \varphi(x)^{-1},((((e \varphi(x)) f) \varphi(y)) g) \varphi(y)^{-1} \varphi(x)^{-1}\right)\right)
\end{aligned}
$$

and all expressions are definable. But we have shown above that

$$
\left(((((e \varphi(x)) f) \varphi(y)) g) \varphi(y)^{-1} \varphi(x)^{-1}\right) \varphi\left(x^{\prime}\right)=((((e \varphi(x)) f) \varphi(y)) g) \varphi(y)^{-1}
$$

and so, by (3),

$$
\begin{gathered}
\begin{array}{r}
y^{\prime} \psi((e \varphi(x)) f, \\
\left.=y \psi((e \varphi(x)) f) \varphi(y)) g) \varphi(y)^{-1} \varphi(x)^{-1} \varphi\left(x^{\prime}\right)\right) \\
=y \psi(f(e \varphi(x)) f) \psi\left(\left((e \varphi(x)) f,((((e \varphi(x)) f) \varphi(y)) g) \varphi(y)^{-1}\right)\right.
\end{array} \\
x^{\prime} \psi\left(((e \varphi(x)) f) \varphi(x)^{-1},((((e \varphi(x)) f) \varphi(y)) g) \varphi(y)^{-1} \varphi(x)^{-1}\right) \\
=x \psi\left(e,((((e \varphi(x)) f) \varphi(y)) g) \varphi(y)^{-1} \varphi(x)^{-1}\right)=x_{1}
\end{gathered}
$$

Thus $z_{1} \lambda\left(y_{1} \lambda\left(x_{1}\right)\right)$ is definable and $(x y) z=z_{1} \lambda\left(y_{1} \lambda\left(x_{1}\right)\right)$.

$7^{\circ}$. If $e, f, g \in E, x \in R_{e}, y \in R_{f}$ and $z \in R_{g}$, then $\left(z_{1} \lambda\left(y_{1}\right)\right) \lambda\left(x_{1}\right)$ is definable and $x(y z)=\left(z_{1} \lambda\left(y_{1}\right)\right) \lambda\left(x_{1}\right)$, where $x_{1}, y_{1}$ and $z_{1}$ have the same meaning as in $6^{\circ}$.

In fact, by $5^{\circ}$ and (10),

$$
y z=(z \psi(g,(f \varphi(y)) g)) \lambda\left(y \psi\left(f,((f \varphi(y)) g) \varphi(y)^{-1}\right)\right)
$$

and the right-hand side is definable. Moreover $e(y z)=((f \varphi(y)) g) \varphi(y)^{-1}$. Again by $5^{\circ}$ and (10), $x(y z)=((y z) \psi(e(y z),(e \varphi(x)) e(y z))) \lambda\left(x \psi\left(e,((e \varphi(x)) e(y z)) \varphi(x)^{-1}\right)\right)$ and the right-hand side is definable. Now $\varphi(y)$ is a one-to-one mapping of $P(f)$ onto $P(f \varphi(y))$ and so, by (4), $\varphi(y)$ is a semilattice isomorphism of $P(f)$ onto $P(f \varphi(y))$. Moreover $(e \varphi(x)) f,((f \varphi(y)) g) \varphi(y)^{-1} \in P(f)$. Hence

$$
\begin{aligned}
\left((e \varphi(x))\left(((f \varphi(y)) g) \varphi(y)^{-1}\right)\right) \varphi(y) & =\left((e \varphi(x)) f\left(((f \varphi(y)) g) \varphi(y)^{-1}\right)\right) \varphi(y) \\
& =(((e \varphi(x)) f) \varphi(y))\left(((f \varphi(y)) g) \varphi(y)^{-1} \varphi(y)\right) \\
& =(((e \varphi(x)) f) \varphi(y))(f \varphi(y)) g=(((e \varphi(x)) f) \varphi(y)) g,
\end{aligned}
$$

since $((e \varphi(x)) f) \varphi(y) \leqq f \varphi(y)$. Therefore

$$
(e \varphi(x)) e(y z)=(e \varphi(x))\left(((f \varphi(y)) g) \varphi(y)^{-1}\right)=((((e \varphi(x)) f) \varphi(y)) g) \varphi(y)^{-1} .
$$

Hence

$x \psi\left(e,((e \varphi(x)) e(y z)) \varphi(x)^{-1}\right)=x \psi\left(e,((((e \varphi(x)) f) \varphi(y)) g) \varphi(y)^{-1} \varphi(x)^{-1}\right)=x_{1}$ 
By $2^{\circ},(6)$ and (3),

$$
\begin{aligned}
&(y z) \psi(e(y z),(e \varphi(x)) e(y z)) \\
&=\left((z \psi(g,(f \varphi(y)) g)) \lambda\left(y \psi\left(f,((f \varphi(y)) g) \varphi(y)^{-1}\right)\right)\right) \\
& \cdot \psi\left(((f \varphi(y)) g) \varphi(y)^{-1},((((e \varphi(x)) f) \varphi(y)) g) \varphi(y)^{-1}\right) \\
&=\left(z \psi\left(g,((((e \varphi(x)) f) \varphi(y)) g) \varphi(y)^{-1} \varphi\left(y \psi\left(f,((f \varphi(y)) g) \varphi(y)^{-1}\right)\right)\right)\right) \\
& \cdot \lambda\left(y \psi(f,((((e \varphi(x)))) \varphi(y)) g) \varphi(y)^{-1}\right)
\end{aligned}
$$

and all expressions are definable. But $y \psi\left(f,((((e \varphi(x)) f) \varphi(y)) g) \varphi(y)^{-1}\right)=y_{1}$ and, by (5),

$$
\begin{gathered}
z \psi\left(g,((((e \varphi(x)) f) \varphi(y)) g) \varphi(y)^{-1} \varphi\left(y \psi\left(f,((f \varphi(y)) g) \varphi(y)^{-1}\right)\right)\right) \\
=z \psi\left(g,((((e \varphi(x)) f) \varphi(y)) g) \varphi(y)^{-1} \varphi(y)\right) \\
=z \psi(g,(((e \varphi(x)) f) \varphi(y)) g)=z_{1} .
\end{gathered}
$$

Hence $\left(z_{1} \lambda\left(y_{1}\right)\right) \lambda\left(x_{1}\right)$ is definable and $x(y z)=\left(z_{1} \lambda\left(y_{1}\right)\right) \lambda\left(x_{1}\right)$.

$8^{\circ}$. $S$ is a semigroup.

In fact, we suppose that $x, y$ and $z$ are elements of $S$ with $e, f, g \in E, x \in R_{e}$, $y \in R_{f}$ and $z \in R_{g}$. Then, by $6^{\circ}$ and $7^{\circ}$, both $z_{1} \lambda\left(y_{1} \lambda\left(x_{1}\right)\right)$ and $\left(z_{1} \lambda\left(y_{1}\right)\right) \lambda\left(x_{1}\right)$ are definable and $(x y) z=z_{1} \lambda\left(y_{1} \lambda\left(x_{1}\right)\right), x(y z)=\left(z_{1} \lambda\left(y_{1}\right)\right) \lambda\left(x_{1}\right)$. The definability implies

$$
e\left(x_{1}\right) \varphi\left(x_{1}\right)=e\left(y_{1}\right), \quad e\left(y_{1}\right) \varphi\left(y_{1}\right)=e\left(z_{1}\right) .
$$

Hence, by (8),

$$
(x y) z=z_{1} \lambda\left(y_{1} \lambda\left(x_{1}\right)\right)=\left(z_{1} \lambda\left(y_{1}\right)\right) \lambda\left(x_{1}\right)=x(y z) .
$$

$9^{\circ}$. If $y \lambda(x)$ is definable, then $y \lambda(x)=x y$.

In fact, by the definability of $y \lambda(x)$, we have $e(y)=e(x) \varphi(x)$. Hence, by $5^{\circ},(10)$ and (2),

$$
\begin{aligned}
x y & =(y \lambda(e(y),(e(x) \varphi(x)) e(y))) \lambda\left(x \psi\left(e(x),((e(x) \varphi(x)) e(y)) \varphi(x)^{-1}\right)\right) \\
& =y \psi(e(y), e(y)) \lambda(x \psi(e(x), e(x)))=y \lambda(x) .
\end{aligned}
$$

$10^{\circ}$. For each $e \in E$, let $e^{*}$ be the element in $R_{e}$ such that $e^{*} \lambda\left(e^{*}\right)$ is definable and $e^{*} \lambda\left(e^{*}\right)=e^{*}$. Then $e^{*}$ is uniquely determined by $e$. Moreover $E^{*}=\left\{e^{*} ; e \in E\right\}$ is the set of all idempotents of $S$.

In fact, by (9), $e^{*}$ is uniquely determined by $e$. We take an arbitrary element $e^{*}$ of $E^{*}$. Then $e^{*} \lambda\left(e^{*}\right)$ is definable. Hence, by $9^{\circ}, e^{* 2}=e^{*} \lambda\left(e^{*}\right)=e^{*}$, and so $e^{*}$ is an idempotent of $S$. Conversely let $x$ be an idempotent of $S$. Then, by $5^{\circ}$ and (10),

$$
x^{2}=(x \psi(e(x),(e(x) \varphi(x)) e(x))) \lambda\left(x \psi\left(e(x),((e(x) \varphi(x)) e(x)) \varphi(x)^{-1}\right)\right)
$$

and the right-hand side is definable. Thus $((e(x) \varphi(x)) e(x)) \varphi(x)^{-1}=e\left(x^{2}\right)=e(x)$ and so $e(x) \varphi(x)=(e(x) \varphi(x)) e(x)$. Therefore $e(x) \varphi(x) \leqq e(x)$. Hence, by (2),

$$
\begin{aligned}
x & =x^{2}=(x \psi(e(x), e(x) \varphi(x))) \lambda(x \psi(e(x), e(x))) \\
& =(x \psi(e(x), e(x) \varphi(x))) \lambda(x) .
\end{aligned}
$$


Hence, by $3^{\circ},(7), 1^{\circ}$ and (5),

$$
\begin{aligned}
e(x) \varphi(x) & =e(x) \varphi((x \psi(e(x), e(x) \varphi(x))) \lambda(x)) \\
& =e(x) \varphi(x) \varphi(x \psi(e(x), e(x) \varphi(x)))=e(x) \varphi(x) \varphi(x) .
\end{aligned}
$$

Therefore $e(x)=e(x) \varphi(x)$. Hence, by (2),

$$
x \psi(e(x), e(x) \varphi(x))=x \psi(2(x), e(x))=x
$$

and so $x \lambda(x)$ is definable and $x=x^{2}=x \lambda(x)$. Therefore $x=e(x)^{*} \in E^{*}$.

$11^{\circ} . \varphi\left(e^{*}\right)$ is the identity mapping of $P(e)$.

In fact, $e^{*} \lambda\left(e^{*}\right)$ is definable and $e^{*}=e^{*} \lambda\left(e^{*}\right)$. Hence, by $3^{\circ}$ and (7), for $f \in P(e)$,

$$
f \varphi\left(e^{*}\right)=f \varphi\left(e^{*} \lambda\left(e^{*}\right)\right)=f \varphi\left(e^{*}\right) \varphi\left(e^{*}\right)
$$

and so $f=f \varphi\left(e^{*}\right)$.

$12^{\circ}$. If $e, g \in E$ and $g \leqq e$, then $e^{*} \psi(e, g)=g^{*}$.

In fact, since $g \leqq e=e\left(e^{*}\right), e^{*} \psi(e, g)$ is definable. Moreover, by $2^{\circ},(6)$ and $11^{\circ}$,

$$
\begin{aligned}
e^{*} \psi(e, g) & =\left(e^{*} \lambda\left(e^{*}\right)\right) \psi(e, g) \\
& =\left(e^{*} \psi\left(e, g \varphi\left(e^{*}\right)\right)\right) \lambda\left(e^{*} \psi(e, g)\right)=\left(e^{*} \psi(e, g)\right) \lambda\left(e^{*} \psi(e, g)\right)
\end{aligned}
$$

and all expressions are definable. Hence $e^{*} \psi(e, g) \in E^{*}$. On the other hand, $e^{*} \psi(e, g) \in R_{g}$ and so $e^{*} \psi(e, g)=g^{*}$.

$13^{\circ}$. The mapping which maps $e$ into $e^{*}$ is a semigroup and semilattice isomorphism of $E$ onto $E^{*}$.

In fact, evidently this mapping is a one-to-one mapping of $E$ onto $E^{*}$. Moreover, by $5^{\circ},(10), 11^{\circ}$ and $12^{\circ}$, for $e, f \in E$,

$$
\begin{aligned}
e^{*} f^{*} & =\left(f^{*} \psi\left(f,\left(e \varphi\left(e^{*}\right)\right) f\right)\right) \lambda\left(e^{*} \psi\left(e,\left(\left(e \varphi\left(e^{*}\right)\right) f\right) \varphi\left(e^{*}\right)^{-1}\right)\right) \\
& =\left(f^{*} \psi(f, e f)\right) \lambda\left(e^{*} \psi(e, e f)\right)=(e f)^{*} \lambda\left((e f)^{*}\right)=(e f)^{*}
\end{aligned}
$$

Hence the mapping is a semigroup isomorphism and so also a semilattice isomorphism of $E$ onto $E^{*}$.

$14^{\circ}$. Let $e, f \in E, x \in R_{e}$ and $f=e \varphi(x)$. We denote the element $y \in R_{f}$ such that $y \lambda(x)=e^{*}$ by $x^{-1}$. Then $x^{-1}$ is uniquely determined by $x$.

Evident from the definition of $\lambda(x)$.

$15^{\circ}$. For every $x \in S, \varphi\left(x^{-1}\right)=\varphi(x)^{-1}$.

In fact, by (4), $\varphi(x)$ is a one-to-one mapping of $P(e(x))$ onto $P(e(x) \varphi(x))$. Hence both $\varphi\left(x^{-1}\right)$ and $\varphi(x)^{-1}$ have the same domain $P(e(x) \varphi(x))$. Let $g \in P(e(x) \varphi(x))$. Then

$$
f=g \varphi(x)^{-1} \in P(e(x)) .
$$

Now, by $11^{\circ}, 14^{\circ}, 3^{\circ}$ and (7),

$$
g \varphi(x)^{-1}=f=f \varphi\left(e(x)^{*}\right)=f \varphi\left(x^{-1} \lambda(x)\right)=f \varphi(x) \varphi\left(x^{-1}\right)=g \varphi\left(x^{-1}\right) .
$$

Hence $\varphi(x)^{-1}=\varphi\left(x^{-1}\right)$. 
$16^{\circ}$. For every $x \in S, x^{-1} x x^{-1}=x^{-1}$.

In fact, by $14^{\circ}$ and $15^{\circ}, e\left(x^{-1}\right) \varphi\left(x^{-1}\right)=e(x) \varphi(x) \varphi(x)^{-1}=e(x)=e\left(e(x)^{*}\right)$. Hence $e(x)^{*} \lambda\left(x^{-1}\right)$ is definable and, by $9^{\circ}, 14^{\circ}$ and $8^{\circ}, e(x)^{*} \lambda\left(x^{-1}\right)=x^{-1} e(x)^{*}=x^{-1}\left(x^{-1} \lambda(x)\right)$ $=x^{-1} x x^{-1}$. Therefore $e\left(x^{-1} x x^{-1}\right)=e\left(e(x)^{*} \lambda\left(x^{-1}\right)\right)=e\left(x^{-1}\right)=e(x) \varphi(x)$. Hence both $\left(x^{-1} x x^{-1}\right) \lambda(x)$ and $x^{-1} \lambda(x)$ are definable and, by $9^{\circ}, 8^{\circ}$ and $14^{\circ}$,

$$
\begin{aligned}
\left(x^{-1} x x^{-1}\right) \lambda(x) & =x x^{-1} x x^{-1}=\left(x^{-1} \lambda(x)\right)\left(x^{-1} \lambda(x)\right) \\
& =e(x)^{*} e(x)^{*}=e(x)^{*}=x^{-1} \lambda(x) .
\end{aligned}
$$

Since $\lambda(x)$ is one-to-one, we have $x^{-1} x x^{-1}=x^{-1}$.

$17^{\circ}$. For every $x \in S, x^{-1} x=(e(x) \varphi(x))^{*}$ and $\left(x^{-1}\right)^{-1}=x$.

In fact, we have shown in the proof of $16^{\circ}$ that $e\left(x^{-1}\right) \varphi\left(x^{-1}\right)=e(x)$. Hence $x \lambda\left(x^{-1}\right)$ is definable and $x \lambda\left(x^{-1}\right)=x^{-1} x$. Therefore $e\left(x^{-1} x\right)=e\left(x \lambda\left(x^{-1}\right)\right)=e\left(x^{-1}\right)$ $=e(x) \varphi(x)$. Now, by $8^{\circ}$ and $16^{\circ},\left(x^{-1} x\right)\left(x^{-1} x\right)=\left(x^{-1} x x^{-1}\right) x=x^{-1} x$. Hence, by $10^{\circ}$, $x^{-1} x \in E^{*}$ and so $x^{-1} x=x \lambda\left(x^{-1}\right)=(e(x) \varphi(x))^{*}$. Therefore, by $14^{\circ}$, we have also $\left(x^{-1}\right)^{-1}=x$.

$18^{\circ}$. For every $x \in S, x x^{-1} x=x$.

In fact, by $17^{\circ}$ and $16^{\circ}, x x^{-1} x=\left(x^{-1}\right)^{-1} x^{-1}\left(x^{-1}\right)^{-1}=\left(x^{-1}\right)^{-1}=x$.

$19^{\circ}$. $S$ is an inverse semigroup and, for each $x \in S, x^{-1}$ is the inverse of $x$.

In fact, by $18^{\circ}, S$ is a regular semigroup and, by $10^{\circ}$ and $13^{\circ}$, two idempotents of $S$ commute with each other. Hence, by [1, Theorem 1.17], $S$ is an inverse semigroup. Moreover, by $16^{\circ}$ and $18^{\circ}, x^{-1}$ is the inverse of $x$.

$20^{\circ}$. For each $e \in E, R_{e}$ is the $\mathscr{R}$-class of $S$ which contains the element $e^{*}$.

In fact, by $18^{\circ}, x \mathscr{R} x x^{-1}$ and $x x^{-1} \in E^{*}$. By [1, Theorem 1.17], each $\mathscr{R}$-class has one and only one idempotent. Hence $x$ is an element in the $\mathscr{R}$-class which contains $e^{*}$ if and only if $x x^{-1}=e^{*}$, if and only if $e(x)=e$ by $14^{\circ}$ and $9^{\circ}$, and so if and only if $x \in R_{e}$.

$21^{\circ}$. If $x \in S, e, f \in E$ and $x \psi(e, f)$ is definable, then $x \psi(e, f)=f^{*} x$.

In fact, since $x \psi(e, f)$ is definable, $x \in R_{e}$ and $f \leqq e$. By $5^{\circ},(10), 11^{\circ},(2), 9^{\circ}, 14^{\circ}$ and $18^{\circ}$,

$$
\begin{aligned}
f^{*} x & =\left(x \psi\left(e,\left(f \varphi\left(f^{*}\right)\right) e\right)\right) \lambda\left(f^{*} \psi\left(f,\left(\left(f \varphi\left(f^{*}\right)\right) e\right) \varphi\left(f^{*}\right)^{-1}\right)\right) \\
& =(x \psi(e, f e)) \lambda\left(f^{*} \psi(f, f e)\right)=(x \psi(e, f)) \lambda\left(f^{*} \psi(f, f)\right) \\
& =(x \psi(e, f)) \lambda\left(f^{*}\right)=f^{*}(x \psi(e, f)) \\
& =(x \psi(e, f))(x \psi(e, f))^{-1}(x \psi(e, f))=x \psi(e, f) .
\end{aligned}
$$

$22^{\circ}$. If $x \in S, e \in E$ and $e \varphi(x)$ is definable, then $(e \varphi(x))^{*}=x^{-1} e^{*} x$.

In fact, since $e \varphi(x)$ is definable, $e \leqq e(x)$. By $19^{\circ}, 17^{\circ}, 21^{\circ}$ and (5),

$$
\begin{aligned}
x^{-1} e^{*} x & =\left(x^{-1} e^{*}\right)\left(e^{*} x\right)=\left(e^{*} x\right)^{-1}\left(e^{*} x\right)=\left(e\left(e^{*} x\right) \varphi\left(e^{*} x\right)\right)^{*} \\
& =(e(x \psi(e(x), e)) \varphi(x \psi(e(x), e)))^{*} \\
& =(e \varphi(x \psi(e(x), e)))^{*}=(e \varphi(x))^{*} .
\end{aligned}
$$

This completes the proof of Theorem 2.2. 
3. A characterization of left ordered inverse semigroups. In this section, we characterize the structure of left ordered inverse semigroups. Theorems 3.4 and 3.6 give a characterization of left ordered inverse semigroups in terms of the ordered commutative idempotent subsemigroup constituted by all idempotents of the inverse semigroup $S$ and the simply ordered $\mathscr{R}$-classes of $S$. Corollaries 3.5 and 3.7 give a characterization in terms of the three mappings $\psi, \varphi$ and $\lambda$.

LEMMA 3.1. A left ordered inverse semigroup $S$ contains no elements of finite order except idempotents.

Proof. By way of contradiction, we assume that $x$ is a nonidempotent element of finite order in $S$. Then we have either $x<x^{2}$ or $x>x^{2}$. If $x<x^{2}$, then $x<x^{2}<\cdots$ $<x^{n}<x^{n+1}=x^{n+2}=\cdots$ for some natural number $n$. If $x>x^{2}$, then $x>x^{2}>\cdots$ $>x^{n}>x^{n+1}=x^{n+2}=\cdots$ for some natural number $n$. In both cases, $y=x^{n}$ is an element of order 2, i.e. $y \neq y^{2}=y^{3}=\cdots$. We put $y^{2}=a$. Since $y^{2}$ and $y y^{-1}$ are idempotents, we have

$$
a y^{-1}=y^{2} y^{-1}=y^{3} y^{-1}=y^{2}\left(y y^{-1}\right)=\left(y y^{-1}\right) y^{2}=y^{2}=a .
$$

Moreover, since $a=y^{2}$ is an idempotent, we have $a=a^{-1}$. First we consider the case when $y y^{-1} \leqq y^{-1} y$. Then

$$
a=a y^{-1}=y^{2} y^{-1}=y\left(y y^{-1}\right) \leqq y\left(y^{-1} y\right)=y .
$$

But $a=y^{2} \neq y$ and so $a<y$. Now we have

$$
\begin{aligned}
\left(y y^{-1}\right)\left(y^{-1} y\right) & =y a^{-1} y=y a y=y^{4}=y^{2}=a<y=\left(y y^{-1}\right) y, \\
y y & =a<y=y\left(y^{-1} y\right) .
\end{aligned}
$$

From the first inequality we obtain $y^{-1} y<y$ and from the second we obtain $y<y^{-1} y$, which is a contradiction. In the case when $y^{-1} y \leqq y y^{-1}$, we obtain a contradiction in a similar way.

LEMMA 3.2. Let $S$ be an inverse semigroup which contains no elements of finite order except idempotents and let $x, y \in S$. Then the following conditions are equivalent to each other:

(a) $y y^{-1} x=x x^{-1} y$;

(b) $y^{-1} x$ is an idempotent ;

(c) $x^{-1} y$ is an idempotent;

(d) $x^{-1} y y^{-1} x=x^{-1} y$;

(e) $y^{-1} x x^{-1} y=y^{-1} x$;

(f) $y^{-1} x=x^{-1} x y^{-1} y$;

(g) $x^{-1} y=x^{-1} x y^{-1} y$;

(h) $x y^{-1} y=y x^{-1} x$;

(i) $x y^{-1}$ is an idempotent;

(j) $y x^{-1}$ is an idempotent ;

(k) $x y^{-1} y x^{-1}=y x^{-1}$; 
(l) $y x^{-1} x y^{-1}=x y^{-1}$;

(m) $x y^{-1}=x x^{-1} y y^{-1}$;

(n) $y x^{-1}=x x^{-1} y y^{-1}$.

Proof. (a) implies (b). In fact, $y^{-1} x=y^{-1}\left(y y^{-1} x\right)=y^{-1} x x^{-1} y=\left(y^{-1} x\right)\left(y^{-1} x\right)^{-1}$ is an idempotent.

(b) implies (c). In fact, since $y^{-1} x$ is an idempotent, $x^{-1} y=\left(y^{-1} x\right)^{-1}=y^{-1} x$ is an idempotent.

(c) implies (d). In fact, $x^{-1} y y^{-1} x=\left(x^{-1} y\right)\left(x^{-1} y\right)^{-1}=\left(x^{-1} y\right)^{2}=x^{-1} y$.

(d) implies (e). In fact, since $x^{-1} y=x^{-1} y y^{-1} x=\left(x^{-1} y\right)\left(x^{-1} y\right)^{-1}$ is an idempotent, $y^{-1} x x^{-1} y=\left(x^{-1} y\right)^{-1}\left(x^{-1} y\right)=\left(x^{-1} y\right)^{2}=x^{-1} y=\left(x^{-1} y\right)^{-1}=y^{-1} x$.

(e) implies (m). In fact, $y^{-1} x=y^{-1} x x^{-1} y$ is an idempotent and so $\left(x y^{-1}\right)^{3}$ $=x\left(y^{-1} x\right)^{2} y^{-1}=x\left(y^{-1} x\right) y^{-1}=\left(x y^{-1}\right)^{2}$. By assumption, $x y^{-1}$ is an idempotent. Moreover, since $y^{-1} x$ is an idempotent, we have $y^{-1} x=\left(y^{-1} x\right)^{-1}=x^{-1} y$. Hence $x y^{-1}=\left(x y^{-1}\right)^{2}=x\left(y^{-1} x\right) y^{-1}=x\left(x^{-1} y\right) y^{-1}=x x^{-1} y y^{-1}$.

(m) implies (n). In fact, $y x^{-1}=\left(x y^{-1}\right)^{-1}=\left(x x^{-1} y y^{-1}\right)^{-1}=x x^{-1} y y^{-1}$.

(n) implies (h). In fact, $y x^{-1}=x x^{-1} y y^{-1}$ is an idempotent and so $y x^{-1}=\left(y x^{-1}\right)^{-1}$ $=x y^{-1}$. Hence $y x^{-1}=\left(y x^{-1}\right)^{2}=\left(y x^{-1}\right)\left(x y^{-1}\right)=y x^{-1} x y^{-1}$. Therefore $x y^{-1} y=$ $\left(x y^{-1}\right) y=\left(y x^{-1}\right) y=\left(y x^{-1} x y^{-1}\right) y=y x^{-1} x$.

By a dual argument, we can prove that (h) implies (i), (i) implies (j), (j) implies (k), (k) implies (l), (l) implies (f), (f) implies (g) and (g) implies (a).

LEMMA 3.3. Let $S$ be an inverse semigroup and let $x, y \in S$ such that $x y^{-1} y$ $=y x^{-1} x$ and $x^{-1} x=y^{-1} y$. Then $x=y$.

Proof. By assumption, we have $x=x x^{-1} x=x y^{-1} y=y x^{-1} x=y y^{-1} y=y$.

THEOREM 3.4. Let $S$ be a left ordered inverse semigroup and let $E$ be the set of all idempotents of $S$. Then $E$ is an ordered commutative idempotent semigroup and, for each $e \in E, R_{e}$ is a simply ordered set with respect to the induced orders of $S$ on $E$ and on $R_{e}$, respectively. Moreover, $S$ satisfies the following conditions:

(11') $S$ contains no elements of finite order except idempotents;

(12') if $e, f \in E, x, y \in R_{e}, x \leqq y$ in $R_{e}$ and $f \leqq e$, then $f x \leqq f y$ in $R_{f}$;

(13') if $e, f \in E, x \in R_{e}, x^{-1} x=f, y, z \in R_{f}$ and $y \leqq z$ in $R_{f}$, then $x y \leqq x z$ in $R_{e}$;

(14') $x \leqq y$ if and only if either

(a) $y y^{-1} x<x x^{-1} y$ in $R_{e f}$, or

(b) $y y^{-1} x=x x^{-1} y$ and $x^{-1} x \leqq y^{-1} y$ in $E$,

where $e$ and $f$ are elements of $E$ such that $x \in R_{e}$ and $y \in R_{f}$.

Proof. It is evident that $E$ is an ordered commutative idempotent semigroup and $R_{e}$ is a simply ordered set with respect to the respective induced orders. The condition ( $\left.11^{\prime}\right)$ is satisfied by Lemma 3.1. If $e, f \in E, x, y \in R_{e}, x \leqq y$ in $R_{e}$ and $f \leqq e$, then

$$
\begin{aligned}
& (f x)(f x)^{-1}=f x x^{-1} f=f e f=f, \\
& (f y)(f y)^{-1}=f y y^{-1} f=f e f=f .
\end{aligned}
$$


Hence $f x, f y \in R_{f}$ and evidently $f x \leqq f y$. Thus we have the condition (12'). If $e, f \in E, x \in R_{e}, x^{-1} x=f, y, z \in R_{f}$ and $y \leqq z$ in $R_{f}$, then

$$
\begin{aligned}
& (x y)(x y)^{-1}=x y y^{-1} x^{-1}=x f x^{-1}=x x^{-1} x x^{-1}=x x^{-1}=e, \\
& (x z)(x z)^{-1}=x z z^{-1} x^{-1}=x f x^{-1}=e .
\end{aligned}
$$

Hence $x y, x z \in R_{e}$ and evidently $x y \leqq x z$. Thus we have the condition (13'). Now we suppose that $x \leqq y$ with $x \in R_{e}$ and $y \in R_{f}$. Then

$$
\begin{aligned}
& \left(y y^{-1} x\right)\left(y y^{-1} x\right)^{-1}=x x^{-1} y y^{-1}=e f, \\
& \left(x x^{-1} y\right)\left(x x^{-1} y\right)^{-1}=x x^{-1} y y^{-1}=e f .
\end{aligned}
$$

Hence $y y^{-1} x, x x^{-1} y \in R_{e f}$. Moreover

$$
y y^{-1} x=x x^{-1} y y^{-1} x \leqq x x^{-1} y y^{-1} y=x x^{-1} y .
$$

If $y y^{-1} x<x x^{-1} y$, then the condition (a) in (14') holds. Next we suppose that $y y^{-1} x=x x^{-1} y$. Then, by Lemma 3.2, $x^{-1} y=x^{-1} x y^{-1} y=y^{-1} x$. Hence $x^{-1} x \leqq x^{-1} y$ $=y^{-1} x \leqq y^{-1} y$ and evidently $x^{-1} x, y^{-1} y \in E$. Therefore the condition (b) in (14') holds. Conversely, if (a) holds, then $x x^{-1} y y^{-1} x=y y^{-1} x<x x^{-1} y=x x^{-1} y y^{-1} y$ and so $x<y$. Next we suppose that (b) holds. By way of contradiction, we assume that $x>y$ is true. Then, by the fact shown above, $x^{-1} x \geqq y^{-1} y$ and so $x^{-1} x=y^{-1} y$. By Lemma 3.2, we have $x y^{-1} y=y x^{-1} x$ and so, by Lemma 3.3, we have $x=y$, which is a contradiction. Thus we have $x \leqq y$.

COROLlary 3.5. Let $S$ be a left ordered inverse semigroup and let $E$ be the set of all idempotents of $S$. Then, in addition to the fact that $S$ satisfies the conclusion of Theorem 2.1, $E$ is an ordered commutative idempotent semigroup and, for each $e \in E$, $R_{e}$ is a simply ordered set with respect to the induced orders of $S$ on $E$ and on $R_{e}$, respectively. Moreover, $S$ satisfies the following conditions:

(11) $S$ contains no elements of finite order except idempotents;

(12) if $e, f \in E, x, y \in R_{e}, x \leqq y$ in $R_{e}$ and $f \leqq e$, then $x \psi(e, f) \leqq y \psi(e, f)$ in $R_{f}$;

(13) if $e, f \in E, x \in R_{e}, e \varphi(x)=f, y, z \in R_{f}$ and $y \leqq z$ in $R_{f}$, then $y \lambda(x) \leqq z \lambda(x)$ in $R_{e}$;

(14) $x \leqq y$ if and only if either

(a) $x \psi(e, e f)<y \psi(f, e f)$ in $R_{e f}$, or

(b) $x \psi(e, e f)=y \psi(f, e f)$ and $e \varphi(x) \leqq f \varphi(y)$ in $E$,

where $e$ and $f$ are elements of $E$ such that $x \in R_{e}$ and $y \in R_{f}$.

THEOREM 3.6. Let $S$ be an inverse semigroup. Suppose that the set $E$ of all idempotents of $S$ is an ordered commutative idempotent semigroup and, for each $e \in E, R_{e}$ is a simply ordered set. Moreover suppose that the conditions $\left(11^{\prime}\right),\left(12^{\prime}\right)$ and $\left(13^{\prime}\right)$ in Theorem 3.4 are satisfied. Then there exists one and only one left ordered inverse semigroup $S$ such that on the set $E$ the order of $S$ coincides with the original order of $E$ and, for each $e \in E$, on the set $R_{e}$ the order of $S$ coincides with the original order of $R_{e}$. This left ordered inverse semigroup $S$ is obtained by defining the order in $S$ by (14') in Theorem 3.4. 
Proof. First we prove that, when we define the order $\leqq$ in $S$ by $\left(14^{\prime}\right), S$ is a left ordered inverse semigroup with the property mentioned in the theorem. We divide the proof into several steps.

$1^{\circ}$. Let $x, y$ be elements of $S$ such that $y y^{-1} x=x x^{-1} y$.

(a) If $x^{-1} x<x^{-1} y y^{-1} x$ in $E$, then $x^{-1} y y^{-1} x \leqq y^{-1} y$ in $E$;

(b) if $x^{-1} y y^{-1} x<x^{-1} x$ in $E$, then $y^{-1} y \leqq x^{-1} y y^{-1} x$ in $E$.

In fact, we suppose that $x^{-1} x<x^{-1} y y^{-1} x$ in $E$. By way of contradiction, we assume that $y^{-1} y<x^{-1} y y^{-1} x$ were true in $E$. By (11'), $S$ contains no elements of finite order except idempotents. Moreover, by assumption, we have $y y^{-1} x=x x^{-1} y$. Hence, by Lemma 3.2, $x^{-1} y y^{-1} x=x^{-1} y=x^{-1} x y^{-1} y$. Therefore $x^{-1} x<x^{-1} x y^{-1} y$ and $y^{-1} y<x^{-1} x y^{-1} y$. But, by Lemma 1.1, $x^{-1} x y^{-1} y$ lies between $x^{-1} x$ and $y^{-1} y$ in $E$, which is a contradiction. Hence $x^{-1} y y^{-1} x \leqq y^{-1} y$ in $E$ and so we obtain (a). We can prove (b) in a similar way.

$2^{\circ}$. The relation $\leqq$ defined in $S$ is really a simple order.

In fact, it is trivial that the relation $\leqq$ in $S$ is reflexive. Now we suppose that $x \leqq y$ and $y \leqq x$ in $S$. Then, by (14'), $y y^{-1} x=x x^{-1} y$ and $x^{-1} x=y^{-1} y$. Hence, by Lemmas 3.2 and 3.3, we have $x=y$ and so the relation $\leqq$ in $S$ is antisymmetric. Now we suppose that $x \leqq y$ and $y \leqq z$ in $S$ with $e, f, g \in E, x \in R_{e}, y \in R_{f}$ and $z \in R_{g}$. Then, by (14'), $y y^{-1} x \leqq x x^{-1} y$ in $R_{e f}$ and $z z^{-1} y \leqq y y^{-1} z$ in $R_{f g}$. Hence, by (12'),

$$
\begin{array}{ll}
y y^{-1} z z^{-1} x=e f g y y^{-1} x \leqq e f g x x^{-1} y=x x^{-1} z z^{-1} y & \text { in } R_{e f g}, \\
x x^{-1} z z^{-1} y=e f g z z^{-1} y \leqq e f g y y^{-1} z=x x^{-1} y y^{-1} z & \text { in } R_{e f g} .
\end{array}
$$

In the case when either $y y^{-1} z z^{-1} x<x x^{-1} z z^{-1} y$ in $R_{e f g}$ or $x x^{-1} z z^{-1} y<x x^{-1} y y^{-1} z$ in $R_{e f g}$, we have $e f g z z^{-1} x=y y^{-1} z z^{-1} x<x x^{-1} y y^{-1} z=e f g x x^{-1} z$ in $R_{e f g}$ and so, by $\left(12^{\prime}\right), z z^{-1} x<x x^{-1} z$ in $R_{e g}$. Hence, by (14'), we have $x \leqq z$. Thus, in what follows, we suppose that $y y^{-1} z z^{-1} x=x x^{-1} z z^{-1} y=x x^{-1} y y^{-1} z$.

(i) The case when $y y^{-1} x<x x^{-1} y$ in $R_{e f}$. We have

$$
\begin{aligned}
x x^{-1} y y^{-1} x & =y y^{-1} x \neq x x^{-1} y=x x^{-1} y y^{-1} y, \\
x x^{-1} y y^{-1} z z^{-1} x & =y y^{-1} z z^{-1} x=x x^{-1} z z^{-1} y=x x^{-1} y y^{-1} z z^{-1} y .
\end{aligned}
$$

Hence $e f g=x x^{-1} y y^{-1} z z^{-1} \neq x x^{-1} y y^{-1}=e f$. Now, in the semilattice $(E, \leqq)$, $e f \leqq f$ and $f g \leqq f$ and so, by Lemma 1.3 , $e f$ and $f g$ are comparable in $(E$, $\leqq$. But, if $e f \leqq f g$ were true, then we would have $e f g=e f$, which is a contradiction. Hence $f g \leqq e f$ and so $e f g=f g$. Similarly we have $e f g=e g$. By assumption, $y \leqq z$ in $S$ and $z z^{-1} y=f g y=e f g y=x x^{-1} z z^{-1} y=x x^{-1} y y^{-1} z=e f g z=f g z=y y^{-1} z$. Hence, by (14'), $y^{-1} y \leqq z^{-1} z$ in $E$. Now, by way of contradiction, we assume that $x \leqq z$ were false in $S$. Then, since $z z^{-1} x=e g x=e f g x=y y^{-1} z z^{-1} x=x x^{-1} y y^{-1} z=e f g z=e g z=x x^{-1} z$, we have, by $\left(14^{\prime}\right), x^{-1} x>z^{-1} z$ in $E$. We put $h=y^{-1} y z^{-1} z \in E$. We have $z z^{-1} y$ $=y y^{-1} z$ and so, by Lemma 3.2, $h=y^{-1} y z^{-1} z=z^{-1} y$. Moreover

$$
\begin{aligned}
h & =z^{-1} y=z^{-1} f g y=z^{-1} e f g y=z^{-1}\left(x x^{-1} z z^{-1} y\right) \\
& =z^{-1}\left(y y^{-1} z z^{-1} x\right)=z^{-1} e f g x=z^{-1} e g x=z^{-1} x .
\end{aligned}
$$


Since $z z^{-1} x=x x^{-1} z$, we have, by Lemma 3.2, $h=z^{-1} x=x^{-1} x z^{-1} z=x^{-1} z$. Hence

$$
h=h^{2}=\left(y^{-1} y z^{-1} z\right)\left(x^{-1} x z^{-1} z\right)=x^{-1} x y^{-1} y z^{-1} z .
$$

Since $y^{-1} y \leqq z^{-1} z<x^{-1} x$ in $E$, we have, by Lemma $1,2, x^{-1} x y^{-1} y \leqq z^{-1} z$ and so $h=x^{-1} x y^{-1} y z^{-1} z=x^{-1} x y^{-1} y$. Hence $x^{-1} z z^{-1} y=\left(x^{-1} z\right)\left(z^{-1} y\right)=h^{2}=h=x^{-1} x y^{-1} y$. Therefore

$$
x y^{-1}=x\left(x^{-1} x y^{-1} y\right) y^{-1}=x\left(x^{-1} z z^{-1} y\right) y^{-1}=x x^{-1} z z^{-1} y y^{-1} \in E .
$$

Hence, by Lemma 3.2, $y y^{-1} x=x x^{-1} y$, which contradicts the assumption that $y y^{-1} x<x x^{-1} y$. Thus we have $x \leqq z$ in $S$.

(ii) The case when $z z^{-1} y<y y^{-1} z$ in $R_{f g}$. In a similar way to (i), we obtain $x \leqq z$.

(iii) The case when $y y^{-1} x \geqq x x^{-1} y$ in $R_{\text {ef }}$ and $z z^{-1} y \geqq y y^{-1} z$ in $R_{f g}$. Since $x \leqq y$ and $y \leqq z$ in $S$, we have, by (14'), $y y^{-1} x=x x^{-1} y, z z^{-1} y=y y^{-1} z$ and $x^{-1} x \leqq y^{-1} y$ $\leqq z^{-1} z$ in $E$. By way of contradiction, we assume that $x x^{-1} z<z z^{-1} x$ in $R_{e g}$. Then, in a similar way to (i), we obtain $z \leqq y$ in $S$, since $x \leqq y$ in $S$. On the other hand, by assumption, $y \leqq z$ in $S$ and so $y=z$, since we have proved that the relation $\leqq$ in $S$ is antisymmetric. Hence

$$
x x^{-1} z=x x^{-1} y=y y^{-1} x=z z^{-1} x,
$$

which is a contradiction. Thus we have $z z^{-1} x \leqq x x^{-1} z$ in $R_{e g}$. If $z z^{-1} x<x x^{-1} z$ in $R_{e g}$, then $x \leqq z$ in $S$ by (14'). Also if $z z^{-1} x=x x^{-1} z$, then $x \leqq z$ in $S$, since $x^{-1} x \leqq z^{-1} z$ in $E$. This completes the proof of the transitivity of the relation $\leqq$.

Now we take arbitrary elements $x, y \in S$. By (14'), if $y y^{-1} x<x x^{-1} y$ in $R_{e f}$, then we have $x \leqq y$ in $S$, while, if $y y^{-1} x>x x^{-1} y$ in $R_{e f}$, then $x \geqq y$ in $S$. Next we suppose that $y y^{-1} x=x x^{-1} y$. Again by (14'), if $x^{-1} x \leqq y^{-1} y$ in $E$, then we have $x \leqq y$ in $S$, while, if $x^{-1} x \geqq y^{-1} y$ in $E$, then $x \geqq y$ in $S$. This completes the proof of $2^{\circ}$.

$3^{\circ}$. With respect to the order $\leqq$ in $S, x \leqq y$ implies $z x \leqq z y$, where $e, f, g \in E$, $x \in R_{e}, y \in R_{f}$ and $z \in R_{g}$.

In fact, we put $x^{\prime}=z x, y^{\prime}=z y, e^{\prime}=z e z^{-1}, f^{\prime}=z f z^{-1}, x^{*}=z e f, y^{*}=z^{-1} z e f x$, $z^{*}=z^{-1} z e f y, e^{*}=z e f z^{-1}$ and $f^{*}=z^{-1} z e f$. Then $e^{\prime}, f^{\prime}, e^{*}, f^{*} \in E$ and

$$
\begin{aligned}
e^{\prime} f^{\prime} & =z e z^{-1} z f z^{-1}=z e f z^{-1}=e^{*}, \\
x^{\prime} x^{\prime-1} & =(z x)(z x)^{-1}=z e z^{-1}=e^{\prime}, \\
y^{\prime} y^{\prime-1} & =(z y)(z y)^{-1}=z f z^{-1}=f^{\prime} .
\end{aligned}
$$

Also we have $x^{\prime} \in R_{e^{\prime}}$ and $y^{\prime} \in R_{f^{\prime}}$. Moreover

$$
\begin{aligned}
& y^{\prime} y^{\prime-1} x^{\prime}=z f z^{-1} z x=(z e f)\left(z^{-1} z e f x\right)=x^{*} y^{*}, \\
& x^{\prime} x^{\prime-1} y^{\prime}=z e z^{-1} z y=(z e f)\left(z^{-1} z e f y\right)=x^{*} z^{*} .
\end{aligned}
$$

Furthermore

$$
\begin{aligned}
& x^{*} x^{*-1}=(z e f)(z e f)^{-1}=z e f z^{-1}=e^{*}, \\
& y^{*} y^{*-1}=\left(z^{-1} z e f x\right)\left(z^{-1} z e f x\right)^{-1}=z^{-1} z e f=f^{*}, \\
& z^{*} z^{*-1}=\left(z^{-1} z e f y\right)\left(z^{-1} z e f y\right)^{-1}=z^{-1} z e f=f^{*}, \\
& x^{*-1} x^{*}=(z e f)^{-1}(z e f)=z^{-1} z e f=f^{*}
\end{aligned}
$$

Also we have $x^{*} \in R_{e^{*}}$ and $y^{*}, z^{*} \in R_{f}$. 
(i) The case when $y y^{-1} x<x x^{-1} y$ in $R_{e f}$. Since $f^{*}=z^{-1} z e f \leqq e f$, we have, by $\left(12^{\prime}\right)$,

$$
y^{*}=\left(z^{-1} z e f\right)\left(y y^{-1} x\right) \leqq\left(z^{-1} z e f\right)\left(x x^{-1} y\right)=z^{*}
$$

in $R_{f *}$. Therefore, since $x^{*} \in R_{e^{*}}$ and $x^{*-1} x^{*}=f^{*}$, we have, by $\left(13^{\prime}\right), y^{\prime} y^{\prime-1} x^{\prime}$ $=x^{*} y^{*} \leqq x^{*} z^{*}=x^{\prime} x^{\prime-1} y^{\prime}$ in $R_{e^{*}}$. If $y^{\prime} y^{\prime-1} x^{\prime}<x^{\prime} x^{\prime-1} y^{\prime}$ in $R_{e^{*}}=R_{e^{\prime} f^{\prime}}$, then, by (14'), $z x=x^{\prime} \leqq y^{\prime}=z y$ in $S$. Next we suppose that $y^{\prime} y^{\prime-1} x^{\prime}=x^{\prime} x^{\prime-1} y^{\prime}$. Then $x^{*} y^{*}$ $=y^{\prime} y^{\prime-1} x^{\prime}=x^{\prime} x^{\prime-1} y^{\prime}=x^{*} z^{*}$. Hence

$$
\begin{aligned}
\left(z^{-1} z e f\right) x & =y^{*}=y^{*} y^{*-1} y^{*}=f^{*} y^{*}=x^{*-1} x^{*} y^{*}=x^{*-1} x^{*} z^{*} \\
& =f^{*} z^{*}=z^{*} z^{*-1} z^{*}=z^{*}=\left(z^{-1} z e f\right) y .
\end{aligned}
$$

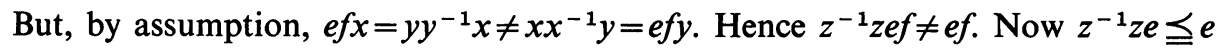
and $e f \leqq e$ and so, by Lemma $1.3, z^{-1} z e$ and $e f$ are comparable in the semilattice $(E, \leqq)$. If $e f \leqq z^{-1} z e$ were true, then we would have $z^{-1} z e f=e f$, which is a contradiction. Hence $z^{-1} z e \leqq e f$ and so $z^{-1} z e f=z^{-1} z e$. Similarly we have $z^{-1} z e f=z^{-1} z f$. Hence

$$
\begin{aligned}
z x & =z\left(z^{-1} z e\right) x=z\left(\left(z^{-1} z e f\right) x\right) \\
& =z\left(\left(z^{-1} z e f\right) y\right)=z\left(z^{-1} z f\right) y=z y .
\end{aligned}
$$

(ii) The case when $y y^{-1} x \geqq x x^{-1} y$ in $R_{e f}$. Since $x \leqq y$ in $S$, we have, by $\left(14^{\prime}\right)$, $y y^{-1} x=x x^{-1} y$ and $x^{-1} x \leqq y^{-1} y$ in $E$. Hence

$$
\begin{aligned}
y^{\prime} y^{\prime-1} x^{\prime} & =x^{*} y^{*}=x^{*} z^{-1} z e f x=\left(x^{*} z^{-1} z\right)\left(y y^{-1} x\right) \\
& =\left(x^{*} z^{-1} z\right)\left(x x^{-1} y\right)=x^{*} z^{-1} z e f y=x^{*} z^{*}=x^{\prime} x^{\prime-1} y^{\prime}
\end{aligned}
$$

Therefore, by Lemma 3.2,

$$
\begin{aligned}
x^{-1} y & =x^{-1} x y^{-1} y=y^{-1} x, \\
x^{\prime-1} y^{\prime} & =x^{\prime-1} x^{\prime} y^{\prime-1} y^{\prime}=y^{\prime-1} x^{\prime} .
\end{aligned}
$$

By way of contradiction, we assume that $x^{-1} y y^{-1} x<x^{-1} x$ is true in $E$. Then, by $1^{\circ}(\mathrm{b}), y^{-1} y \leqq x^{-1} y y^{-1} x<x^{-1} x$ in $E$, which is a contradiction. Hence $x^{-1} x$ $\leqq x^{-1} y y^{-1} x$ in $E$. Similarly we have $y^{-1} x x^{-1} y \leqq y^{-1} y$ in $E$. Hence, in $E$,

$$
\begin{aligned}
x^{-1} x^{\prime} & =(z x)^{-1}(z x)=(z x)^{-1}(z x)\left(x^{-1} x\right) \leqq(z x)^{-1}(z x)\left(x^{-1} y y^{-1} x\right) \\
& =(z x)^{-1} z y y^{-1} x=(z x)^{-1}(z y)\left(y^{-1} x\right)=\left(x^{\prime-1} y^{\prime}\right)\left(y^{-1} x\right) \\
& =\left(y^{-1} x^{\prime}\right)\left(x^{-1} y\right)=(z y)^{-1}(z x)\left(x^{-1} y\right)=(z y)^{-1} z x x^{-1} y \\
& =(z y)^{-1}(z y)\left(y^{-1} x x^{-1} y\right) \leqq(z y)^{-1}(z y)\left(y^{-1} y\right)=(z y)^{-1}(z y) \\
& =y^{\prime-1} y^{\prime} .
\end{aligned}
$$

Therefore, by $\left(14^{\prime}\right)$, we have $z x=x^{\prime} \leqq y^{\prime}=z y$ in $S$. This completes the proof of $3^{\circ}$.

$4^{\circ}$. On the set $E$ the order of $S$ coincides with the original order of $E$.

In fact, for $e, f \in E, f f^{-1} e=e f=e e^{-1} f$ and $e^{-1} e=e, f^{-1} f=f$. Hence, by $\left(14^{\prime}\right)$, $e \leqq f$ with respect to the order in $S$ if and only if $e \leqq f$ with respect to the order in $E$.

$5^{\circ}$. For each $e \in E$, on the set $R_{e}$ the order of $S$ coincides with the original order of $R_{e}$. 
In fact, for $x, y \in R_{e}, y y^{-1} x=x x^{-1} x=x$ and $x x^{-1} y=y y^{-1} y=y$. Hence, by (14'), $x<y$ with respect to the order of $S$ if and only if $x<y$ with respect to the order of $R_{e}$.

This completes the proof of the fact that, when we define the order in $S$ by $\left(14^{\prime}\right)$, $S$ is a left ordered inverse semigroup with the property mentioned in the theorem. The uniqueness of such left ordered inverse semigroups is almost trivial by Theorem 3.4.

COROLlaRY 3.7. In addition to the assumption of Theorem 2.2, we suppose that $E$ is an ordered commutative idempotent semigroup, that, for each $e \in E, R_{e}$ is a simply ordered set and that the conditions (11), (12) and (13) in Corollary 3.5 are satisfied. We define the product in $S$ by (10) in Theorem 2.1 and the order in $S$ by (14) in Corollary 3.5. Then $S$ is a left ordered inverse semigroup such that the semigroup and semilattice isomorphism of $E$ onto $E^{*}$ in Theorem 2.2 which maps $e$ into $e^{*}$ is an order isomorphism of the ordered semigroup $E$ onto the ordered semigroup $E^{*}$ induced by the order of $S$ and moreover, for each $e \in E$, the order induced in $R_{e}$ by the order of $S$ coincides with the original order in $R_{e}$.

4. Some properties of left ordered inverse semigroups. In this section, we give some properties of left ordered inverse semigroups which we need in the following sections.

LEMMA 4.1. Let $x, y$ be elements of a left ordered inverse semigroup $S$. Then

(a) $y y^{-1} x<x x^{-1} y$ if and only if $x y^{-1} y<y x^{-1} x$;

(b) $y y^{-1} x>x x^{-1} y$ if and only if $x y^{-1} y>y x^{-1} x$;

(c) $y y^{-1} x=x x^{-1} y$ if and only if $x y^{-1} y=y x^{-1} x$.

Proof. We put $x^{\prime}=x y^{-1} y$ and $y^{\prime}=y x^{-1} x$. Then

$$
\begin{aligned}
y^{\prime} y^{\prime-1} x^{\prime} & =y x^{-1} x y^{-1} x y^{-1} y=y x^{-1} x\left(y^{-1} x x^{-1} y y^{-1} x\right)\left(y^{-1} y\right) \\
& =y x^{-1} x y^{-1} x\left(x^{-1} y y^{-1} x\right)\left(y^{-1} y\right)=y x^{-1} x y^{-1} x\left(y^{-1} y\right)\left(x^{-1} y y^{-1} x\right) \\
& =\left(y x^{-1} x y^{-1}\right)\left(x y^{-1} y x^{-1}\right)\left(y y^{-1} x\right)
\end{aligned}
$$

and similarly $x^{\prime} x^{\prime-1} y^{\prime}=\left(x y^{-1} y x^{-1}\right)\left(y x^{-1} x y^{-1}\right)\left(x x^{-1} y\right)$. First we suppose that $y y^{-1} x<x x^{-1} y$. Then

$$
\begin{aligned}
y^{\prime} y^{\prime-1} x^{\prime} & =\left(y x^{-1} x y^{-1}\right)\left(x y^{-1} y x^{-1}\right)\left(y y^{-1} x\right) \\
& =\left(x y^{-1} y x^{-1}\right)\left(y x^{-1} x y^{-1}\right)\left(y y^{-1} x\right) \\
& \leqq\left(x y^{-1} y x^{-1}\right)\left(y x^{-1} x y^{-1}\right)\left(x x^{-1} y\right)=x^{\prime} x^{\prime-1} y^{\prime}
\end{aligned}
$$

By way of contradiction, we assume that $y^{\prime} y^{\prime-1} x^{\prime}=x^{\prime} x^{-1} y^{\prime}$ is true. Then, by Lemma 3.2, $x^{-1} x y^{-1} x y^{-1} y=y^{-1} x^{\prime}=x^{\prime-1} x^{\prime} y^{-1} y^{\prime}=x^{-1} y^{\prime}=y^{-1} y x^{-1} y x^{-1} x$. Hence

$$
\begin{aligned}
x y^{-1} x y^{-1} & =x\left(x^{-1} x y^{-1} x y^{-1} y\right) y^{-1}=x\left(y^{-1} y x^{-1} y x^{-1} x\right) y^{-1} \\
& =\left(x y^{-1} y x^{-1}\right)\left(y x^{-1} x y^{-1}\right) .
\end{aligned}
$$


Therefore $\left(x y^{-1}\right)^{2}$ is an idempotent and so $x y^{-1}$ is an element of finite order. By Lemma 3.1, $x y^{-1}$ is an idempotent and so, by Lemma 3.2,yy $x=x x^{-1} y$, which is a contradiction. Therefore $x^{\prime} x^{\prime-1} y^{\prime} y^{\prime-1} x^{\prime}=y^{\prime} y^{\prime-1} x^{\prime}<x^{\prime} x^{\prime-1} y^{\prime}=x^{\prime} x^{\prime-1} y^{\prime} y^{\prime-1} y^{\prime}$ and so $x y^{-1} y=x^{\prime}<y^{\prime}=y x^{-1} x$. Thus we have proved that $y y^{-1} x<x x^{-1} y$ implies $x y^{-1} y$ $<y x^{-1} x$. In a similar way we can prove that $y y^{-1} x>x x^{-1} y$ implies $x y^{-1} y>y x^{-1} x$. The assertion (c) is contained in Lemma 3.2. Hence, conversely, $x y^{-1} y<y x^{-1} x$ implies $y y^{-1} x<x x^{-1} y$ and $x y^{-1} y>y x^{-1} x$ implies $y y^{-1} x>x x^{-1} y$. This completes the proof of Lemma 4.1.

LEMMA 4.2. Let $x, y$ be elements of a left ordered inverse semigroup $S$ which are $\mathscr{R}$-equivalent or $\mathscr{L}$-equivalent to one another. Then

(a) $y y^{-1} x<x x^{-1} y$ if and only if $x<y$;

(b) $y y^{-1} x>x x^{-1} y$ if and only if $x>y$;

(c) $y y^{-1} x=x x^{-1} y$ if and only if $x=y$.

Proof. If $x \mathscr{R} y$, then we have $x x^{-1}=y y^{-1}$. Hence $x=x x^{-1} x=y y^{-1} x, y=y y^{-1} y$ $=x x^{-1} y$. Therefore we obtain the conclusion trivially. If $x \mathscr{L} y$, then $x^{-1} x=y^{-1} y$ and so $x=x x^{-1} x=x y^{-1} y, y=y y^{-1} y=y x^{-1} x$. Hence we obtain the conclusion by Lemma 4.1.

LEMMA 4.3. The following conditions for an element $x$ of a left ordered inverse semigroup $S$ are equivalent:

(a) $x$ is positive;

(b) $x^{-1} x<x$;

(c) $x x^{-1}<x$.

Proof. (a) implies (b). In fact, $x x^{-1} x=x<x^{2}$ and so $x^{-1} x<x$.

(b) implies (c). In fact, $\left(x^{-1} x\right) x^{-1} x=x^{-1} x<x=x x^{-1} x x^{-1} x=x\left(x^{-1} x\right)^{-1}\left(x^{-1} x\right)$. Hence, by Lemma 4.1, $\left(x^{-1} x\right) x x^{-1}=x x^{-1}\left(x^{-1} x\right)<\left(x^{-1} x\right)\left(x^{-1} x\right)^{-1} x=\left(x^{-1} x\right) x$. Therefore $x x^{-1}<x$.

(c) implies (a). In fact, $x x^{-1}\left(x^{-1} x\right)=\left(x^{-1} x\right) x x^{-1} \leqq\left(x^{-1} x\right) x=\left(x^{-1} x\right)\left(x^{-1} x\right)^{-1} x$. Hence, by Lemma 4.1, $x^{-1} x=\left(x^{-1} x\right) x^{-1} x \leqq x\left(x^{-1} x\right)^{-1}\left(x^{-1} x\right)=x x^{-1} x x^{-1} x=x$. Therefore $x=x x^{-1} x \leqq x^{2}$. But, if $x=x^{2}$, then $x$ is an idempotent and so $x x^{-1}=x^{2}$ $=x$, contradicting the assumption. Hence $x$ is positive.

LEMMA 4.3'. The following conditions for an element $x$ of a left ordered inverse semigroup $S$ are equivalent:

(a) $x$ is nonpositive;

(b) $x \leqq x^{-1} x$;

(c) $x \leqq x x^{-1}$.

As the order dual of Lemma 4.3, we have

LEMMA 4.4. The following conditions for an element $x$ of a left ordered inverse semigroup $S$ are equivalent:

(a) $x$ is negative; 
(b) $x<x^{-1} x$;

(c) $x<x x^{-1}$.

Lemma 4.4'. The following conditions for an element $x$ of a left ordered inverse semigroup $S$ are equivalent:

(a) $x$ is nonnegative;

(b) $x^{-1} x \leqq x$;

(c) $x x^{-1} \leqq x$.

LEMMA 4.5. Let $x$ be an element of a left ordered inverse semigroup $S$. Then $x$ is positive if and only if $x^{-1}$ is negative.

Proof. By Lemma 4.3, $x$ is positive if and only if $x x^{-1}<x$. If $x x^{-1}<x$, then $x x^{-1}<x x^{-1} x$ and so $x^{-1}<x^{-1} x$. Conversely, if $x^{-1}<x^{-1} x$, then $x^{-1} x x^{-1}<x^{-1} x$ and so $x x^{-1}<x$. Thus $x x^{-1}<x$ if and only if $x^{-1}<x^{-1} x$ and so, by Lemma 4.4, if and only if $x^{-1}$ is negative.

LEMMA 4.6. Let $x, y$ be nonnegative elements of a left ordered inverse semigroup $S$. Then $x y$ is nonnegative.

Proof. Since $x$ is nonnegative, $x^{-1}$ is nonpositive by Lemma 4.5. Hence, by Lemma $4.3^{\prime}, x^{-1} \leqq x^{-1} x$. Therefore $(x y)(x y)^{-1}=x y y^{-1} x^{-1} \leqq x y y^{-1} x^{-1} x=x y y^{-1}$. Since $y$ is nonnegative, we have $y y^{-1} \leqq y$ by Lemma $4.4^{\prime}$. Hence $(x y)(x y)^{-1}$ $\leqq x y y^{-1} \leqq x y$. Hence, by Lemma $4.4^{\prime}, x y$ is nonnegative.

As the order dual of Lemma 4.6, we have

LEMMA 4.7. Let $x, y$ be nonpositive elements of a left ordered inverse semigroup $S$. Then $x y$ is nonpositive.

By Lemma 4.6, the set $\boldsymbol{P}$ of all nonnegative elements of a left ordered inverse semigroup $S$ forms a subsemigroup of $S$, which is called the nonnegative part of $S$. Also, by Lemma 4.7 , the set $Q$ of all nonpositive elements of $S$ forms a subsemigroup of $S$, which is called the nonpositive part of $S$.

Lemma 4.8. Let $x, y$ be elements of a left ordered inverse semigroup $S$. Then the following conditions are equivalent:

(a) $y y^{-1} x<x x^{-1} y$;

(b) $y^{-1} y x^{-1} y x^{-1} x$ is positive;

(c) $x^{-1} y$ is positive.

Proof. (a) implies (b). In fact, we put $x^{\prime}=x y^{-1} y$ and $y^{\prime}=y x^{-1} x$. Then, by Lemma 4.1, $x^{\prime}=x y^{-1} y<y x^{-1} x=y^{\prime}$ and also $x^{\prime-1} x^{\prime}=x^{-1} x y^{-1} y=y^{-1} y^{\prime}$. Hence $x^{\prime-1} y^{\prime}=x^{\prime-1} y^{\prime} y^{\prime-1} y^{\prime}=x^{-1} y^{\prime} x^{\prime-1} x^{\prime} \leqq x^{\prime-1} y^{\prime} x^{\prime-1} y^{\prime}=\left(x^{\prime-1} y^{\prime}\right)^{2}$. By way of contradiction, we assume that $x^{\prime-1} y^{\prime}=\left(x^{\prime-1} y^{\prime}\right)^{2}$ is true. Then $x^{\prime-1} y^{\prime}$ is an idempotent and so, by Lemma 3.2, $y^{\prime} y^{\prime-1} x^{\prime}=x^{\prime} x^{\prime-1} y^{\prime}$. Now $x^{\prime} \mathscr{L} x^{\prime-1} x^{\prime}=y^{\prime-1} y^{\prime} \mathscr{L} y^{\prime}$ and so $x^{\prime}$ and $y^{\prime}$ are $\mathscr{L}$-equivalent. Hence, by Lemma $4.2, x^{\prime}=y^{\prime}$, which contradicts the fact that $x^{\prime}<y^{\prime}$. Hence $x^{\prime-1} y^{\prime}<\left(x^{\prime-1} y^{\prime}\right)^{2}$ and so $y^{-1} y x^{-1} y x^{-1} x=x^{-1} y^{\prime}$ is positive. 
(b) implies (c). In fact, by way of contradiction, we assume that $x^{-1} y$ is nonpositive. Then, by Lemma $4.7, y^{-1} y x^{-1} y x^{-1} x$ is also nonpositive, which contradicts the assumption. Thus $x^{-1} y$ is positive.

(c) implies (a). In fact, by Lemma 4.3, $\left(x^{-1} y y^{-1}\right)\left(y y^{-1} x\right)=\left(x^{-1} y\right)\left(x^{-1} y\right)^{-1}$ $<x^{-1} y=\left(x^{-1} y y^{-1}\right)\left(x x^{-1} y\right)$ and so $y y^{-1} x<x x^{-1} y$.

Lemma 4.9. Let $x, y$ be elements of a left ordered inverse semigroup $S$ which are $\mathscr{R}$-equivalent or $\mathscr{L}$-equivalent to one another. Then $x<y$ if and only if $x^{-1} y$ is positive.

Proof. This lemma follows immediately from Lemmas 4.2 and 4.8.

LemMA 4.10. Let $x, y, z$ be elements of a left ordered inverse semigroup $S$. If $x y^{-1} y \neq y x^{-1} x$ and $x y^{-1} y z=y x^{-1} x z$, then $x z=y z$.

Proof. By assumption,

$$
\begin{aligned}
x\left(x^{-1} x y^{-1} y\right) & =x y^{-1} y \neq y x^{-1} x=y\left(x^{-1} x y^{-1} y\right) \\
x\left(x^{-1} x y^{-1} y z z^{-1}\right) & =\left(x y^{-1} y z\right) z^{-1}=\left(y x^{-1} x z\right) z^{-1}=y\left(x^{-1} x y^{-1} y z z^{-1}\right) .
\end{aligned}
$$

Hence $x^{-1} x y^{-1} y \neq x^{-1} x y^{-1} y z z^{-1}$. Now $\left(x^{-1} x\right)\left(y^{-1} y\right) \leqq x^{-1} x,\left(x^{-1} x\right)\left(z z^{-1}\right) \leqq x^{-1} x$ and so, by Lemma 1.3, $\left(x^{-1} x\right)\left(y^{-1} y\right)$ and $\left(x^{-1} x\right)\left(z z^{-1}\right)$ are comparable in the semilattice $(E, \preceq)$. Since $x^{-1} x y^{-1} y \neq x^{-1} x y^{-1} y z z^{-1}$, we have $\left(x^{-1} x\right)\left(z z^{-1}\right)$ $\leqq\left(x^{-1} x\right)\left(y^{-1} y\right)$ and so $x^{-1} x z z^{-1}=x^{-1} x y^{-1} y z z^{-1}$. Similarly we have $y^{-1} y z z^{-1}$ $=x^{-1} x y^{-1} y z z^{-1}$. Hence

$$
\begin{aligned}
x z & =x\left(x^{-1} x z z^{-1}\right) z=x\left(x^{-1} x y^{-1} y z z^{-1}\right) z=x y^{-1} y z=y x^{-1} x z \\
& =y\left(x^{-1} x y^{-1} y z z^{-1}\right) z=y\left(y^{-1} y z z^{-1}\right) z=y z .
\end{aligned}
$$

5. A characterization of ordered inverse semigroups. In this section, we characterize the structure of ordered inverse semigroups. Theorem 5.4 gives a condition in order that a left ordered inverse semigroup is an ordered inverse semigroup. Corollary 5.5 gives a characterization of ordered inverse semigroups in terms of the three mappings $\psi, \varphi$ and $\lambda$.

LEMMA 5.1. Let $S$ be a left ordered inverse semigroup and let $E$ be the set of all idempotents of $S$. Then $S$ satisfies the condition

(12R) if $e, f \in E, x, y \in L_{e}, x \leqq y$ in $L_{e}$ and $f \leqq e$, then $x f \leqq y f$ in $L_{f}$.

Proof. We put $x^{\prime}=x f$ and $y^{\prime}=y f$. Since $x \mathscr{L} y$ and $x \leqq y$, we have, by Lemma 4.2, $y y^{-1} x \leqq x x^{-1} y$. Hence

$$
\begin{aligned}
y^{\prime} y^{-1} x^{\prime} & =(y f)(y f)^{-1}(x f)=y f y^{-1} x f=y f y^{-1} x\left(x^{-1} y y^{-1} x\right) f \\
& =y f y^{-1} x f x^{-1} y y^{-1} x \leqq y f y^{-1} x f x^{-1} x x^{-1} y=\left(x f x^{-1}\right)\left(y f y^{-1}\right) x x^{-1} y \\
& =x f x^{-1} y\left(y^{-1} x x^{-1} y\right) f=x f x^{-1} y f=(x f)(x f)^{-1}(y f) \\
& =x^{\prime} x^{\prime-1} y^{\prime} .
\end{aligned}
$$


Since $x \mathscr{L} y$, we have $x^{\prime}=x f \mathscr{L} y f=y^{\prime}$. Hence, by Lemma 4.2, $x f=x^{\prime} \leqq y^{\prime}=y f$.

LEMMA 5.2. Let $(S, \leqq)$ be a left ordered inverse semigroup and let $E$ be the set of all idempotents of $S$. Suppose that $(S, \leqq)$ satisfies the condition

(13R) if $e, f \in E, x \in L_{e}, x x^{-1}=f, y, z \in L_{f}$ and $y \leqq z$ in $L_{f}$, then $y x \leqq z x$ in $L_{e}$. Then there exists one and only one right ordered inverse semigroup $\left(S, \leqq_{1}\right)$ such that the order $\varliminf_{1}$ coincides with the order $\leqq$ on the set $E$ and also on the set $L_{e}$ for each $e \in E$.

Proof. This lemma follows immediately from Theorem 3.4, Lemma 5.1 and the left-right dual of Theorem 3.6.

The right ordered inverse semigroup $\left(S, \leqq_{1}\right)$ in Lemma 5.2 is called the associated right ordered semigroup of the left ordered inverse semigroup $(S, \leqq)$.

LEMMA 5.3. Let $S$ be a left ordered inverse semigroup and let $E$ be the set of all idempotents of $S$. Then the condition

(14R) $x \leqq y$ if and only if either

(a) $x y^{-1} y<y x^{-1} x$ in $L_{e f}$, or

(b) $x y^{-1} y=y x^{-1} x$ and $x x^{-1} \leqq y y^{-1}$ in $E$,

where $e$ and $f$ are elements of $E$ such that $x \in L_{e}$ and $y \in L_{f}$, is equivalent to the condition

(16') if $e, f, g \in E, x \in R_{e}, f \leqq e, g \leqq e$ and $f \leqq g$, then $x^{-1} f x \leqq x^{-1} g x$.

Proof. (14R) implies (16'). In fact, we suppose that $e, f, g \in E, x \in R_{e}, f \leqq e$, $g \leqq e$ and $f \leqq g$. Then $x^{-1} f \leqq x^{-1} g$. Now $\left(x^{-1} f\right)\left(x^{-1} g\right)^{-1}\left(x^{-1} g\right)=x^{-1} f g$ $=\left(x^{-1} g\right)\left(x^{-1} f\right)^{-1}\left(x^{-1} f\right)$. Hence, by $(14 \mathrm{R}), x^{-1} f x=\left(x^{-1} f\right)\left(x^{-1} f\right)^{-1} \leqq\left(x^{-1} g\right)\left(x^{-1} g\right)^{-1}$ $=x^{-1} g x$.

(16') implies (14R). In fact, by Theorem $3.4, x \leqq y$ if and only if either

$\left(\mathrm{a}^{*}\right) y y^{-1} x<x x^{-1} y$ or

(b*) $y y^{-1} x=x x^{-1} y$ and $x^{-1} x \leqq y^{-1} y$.

By Lemma 4.1, (a) is equivalent to ( $\left.\mathrm{a}^{*}\right)$ and also the first condition of (b) is equivalent to the first condition of $\left(\mathrm{b}^{*}\right)$. Hence, in what follows, we consider the case when $x y^{-1} y=y x^{-1} x$ and $y y^{-1} x=x x^{-1} y$. First we suppose that $x x^{-1}<y y^{-1}$. By Lemma 1.1, $x x^{-1} \leqq x x^{-1} y y^{-1} \leqq y y^{-1}$. Hence, by $\left(16^{\prime}\right)$,

$$
\begin{aligned}
x^{-1} x & =x^{-1}\left(x x^{-1}\right) x \leqq x^{-1}\left(x x^{-1} y y^{-1}\right) x=x^{-1} y y^{-1} x, \\
y^{-1} x x^{-1} y & =y^{-1}\left(x x^{-1} y y^{-1}\right) y \leqq y^{-1}\left(y y^{-1}\right) y=y^{-1} y .
\end{aligned}
$$

Since $x y^{-1} y=y x^{-1} x$, we have, by Lemma 3.2, $x^{-1} y y^{-1} x=x^{-1} y=x^{-1} x y^{-1} y$ $=y^{-1} x=y^{-1} x x^{-1} y$, and so $x^{-1} x \leqq x^{-1} y y^{-1} x=y^{-1} x x^{-1} y \leqq y^{-1} y$. But, if $x^{-1} x$ $=y^{-1} y$, then, by Lemma 3.3, we have $x=y$, which contradicts the fact that $x x^{-1}$ $<y y^{-1}$. Thus we have proved that $x x^{-1}<y y^{-1}$ implies $x^{-1} x<y^{-1} y$. Similarly we can prove that $y y^{-1}<x x^{-1}$ implies $y^{-1} y<x^{-1} x$. Finally, if $x x^{-1}=y y^{-1}$, then, by 
Lemma 4.2, we have $x=y$ and so $x^{-1} x=y^{-1} y$. Hence

$$
\begin{array}{lll}
x x^{-1}<y y^{-1} & \text { if and only if } & x^{-1} x<y^{-1} y \\
x x^{-1}>y y^{-1} & \text { if and only if } & x^{-1} x>y^{-1} y \\
x x^{-1}=y y^{-1} & \text { if and only if } & x^{-1} x=y^{-1} y .
\end{array}
$$

Therefore (b) is equivalent to $\left(b^{*}\right)$. Hence (14R) holds.

THEOREM 5.4. Let $S$ be a left ordered inverse semigroup and let $E$ be the set of all idempotents of $S$. In order that $S$ is an ordered inverse semigroup, it is necessary and sufficient that it satisfies the following conditions:

(15') if $x x^{-1}=y^{-1} y=z^{-1} z$ and $y \leqq z$, then $y x \leqq z x$;

$\left(16^{\prime}\right)$ if $e, f, g \in E, x \in R_{e}, f \leqq e, g \leqq e$ and $f \leqq g$, then $x^{-1} f x \leqq x^{-1} g x$.

Proof. The necessity of these conditions are trivial. We prove the sufficiency and suppose that $S$ satisfies the conditions $\left(15^{\prime}\right)$ and $\left(16^{\prime}\right)$. The condition $\left(15^{\prime}\right)$ is nothing but the condition (13R) and so, by Lemma 5.2, there exists the associated right ordered semigroup $\left(S, \varliminf_{1}\right)$ of the original left ordered inverse semigroup $(S, \leqq)$. By the left-right dual of Theorem 3.6 and Lemma 5.3, the condition (16') means that the order $\varliminf_{1}$ coincides with the original order $\leqq$. Thus $S$ is an ordered inverse semigroup.

COROLlaRY 5.5. Let $S$ be an ordered inverse semigroup and let $E$ be the set of all idempotents of $S$. Then, in addition to the fact that $S$ satisfies the conclusion of Corollary 3.5, $S$ satisfies the following conditions:

(15) if $e, f, g \in E, x \in R_{e}, y \in R_{f}, z \in R_{g}, e=f \varphi(y)=g \varphi(z)$ and $y \psi(f, f g) \leqq z \psi(g, f g)$ in $R_{f g}$, then $y x \psi(f, f g) \leqq z x \psi(g, f g)$ in $R_{f g}$;

(16) if $e, f, g \in E, x \in R_{e}, f \leqq e, g \leqq e$ and $f \leqq g$ in $E$, then $f \varphi(x) \leqq g \varphi(x)$ in $E$.

Conversely, in addition to the assumption of Corollary 3.7, we suppose that the conditions (15) and (16) are satisfied. We define the product in $S$ by (10) in Theorem 2.1 and the order in $S$ by (14) in Corollary 3.5. Then $S$ is an ordered inverse semigroup.

THEOREM 5.6. Let $S$ be a left ordered inverse semigroup. Then each one of the following conditions is equivalent to the condition $\left(15^{\prime}\right)$ in Theorem 5.4:

(15a) if $x y^{-1} y<y x^{-1} x$, then $y x^{-1}$ is positive;

(15b) if $x \mathscr{L} y$ and $x<y$, then $y x^{-1}$ is positive;

(15c) if $x \mathscr{R} y$ and $x<y$, then $y x^{-1}$ is positive;

(15d) if $x \mathscr{L} y$ and $y x^{-1}$ is positive, then $x<y$;

(15e) if $x \mathscr{R} y$ and $y x^{-1}$ is positive, then $x<y$;

(15f) if $y y^{-1} x<x x^{-1} y$, then $x^{-1} x y^{-1}<y^{-1} y x^{-1}$;

$(15 \mathrm{~g})$ if $x \mathscr{L} y$ and $x<y$, then $y^{-1}<x^{-1}$;

(15h) if $x \mathscr{R} y$ and $x<y$, then $y^{-1}<x^{-1}$;

(15i) if $x y$ is positive, then $y x$ is positive;

(15j) if $x y^{-1} y$ is positive, then $y x y^{-1}$ is positive;

(15k) if $y y^{-1} x$ is positive, then $y^{-1} x y$ is positive; 
(15l) if $y$ is positive, then $x^{-1} y x$ is nonnegative for every $x \in S$;

(15m) if $x^{-1} y$ is not idempotent and $x<y$, then $x z \leqq y z$ for every $z \in S$.

Proof. (15') implies (15a). In fact, we suppose that $x y^{-1} y<y x^{-1} x$. We have $\left(y^{-1} y x^{-1}\right)\left(y^{-1} y x^{-1}\right)^{-1}=x^{-1} x y^{-1} y=\left(x y^{-1} y\right)^{-1}\left(x y^{-1} y\right)=\left(y x^{-1} x\right)^{-1}\left(y x^{-1} x\right)$. Hence, by $\left(15^{\prime}\right)$,

$$
\begin{aligned}
\left(y x^{-1}\right)^{-1}\left(y x^{-1}\right) & =x y^{-1} y x^{-1}=\left(x y^{-1} y\right)\left(y^{-1} y x^{-1}\right) \\
& \leqq\left(y x^{-1} x\right)\left(y^{-1} y x^{-1}\right)=y x^{-1} .
\end{aligned}
$$

But, if $\left(y x^{-1}\right)^{-1}\left(y x^{-1}\right)=y x^{-1}$ were true, then $y x^{-1}$ is an idempotent and so, by Lemma 3.2, we have $x y^{-1} y=y x^{-1} x$, which is a contradiction. Hence $\left(y x^{-1}\right)^{-1}\left(y x^{-1}\right)$ $<y x^{-1}$ and so, by Lemma $4.3, y x^{-1}$ is positive.

(15a) implies (15c). In fact, we suppose that $x \mathscr{R} y$ and $x<y$. Then, by Lemma 4.2, we have $y y^{-1} x<x x^{-1} y$. Hence, by Lemma 4.1, $x y^{-1} y<y x^{-1} x$ and so, by (15a), $y x^{-1}$ is positive.

(15c) implies (15e). In fact, we suppose that $x \mathscr{R} y$ and $y x^{-1}$ is positive. By way of contradiction, we assume that $y \leqq x$. If $y<x$, then, by (15c), $x y^{-1}$ is positive and so, by Lemma $4.5, y x^{-1}=\left(x y^{-1}\right)^{-1}$ is negative, which is a contradiction. If $y=x$, then $y x^{-1}=x x^{-1}$ is an idempotent, which is also a contradiction. Thus we have $x<y$.

(15e) implies (15f). In fact, we suppose that $y y^{-1} x<x x^{-1} y$. Then, by Lemma 4.8, $y^{-1} y x^{-1} y x^{-1} x$ is positive. Now $x^{-1} x y^{-1} \mathscr{R} x^{-1} x y^{-1} y \mathscr{R} y^{-1} y x^{-1}$ and moreover $\left(y^{-1} y x^{-1}\right)\left(x^{-1} x y^{-1}\right)^{-1}=y^{-1} y x^{-1} y x^{-1} x$ is positive. Hence, by (15e), $x^{-1} x y^{-1}$ $<y^{-1} y x^{-1}$.

(15f) implies (15h). In fact, we suppose that $x \mathscr{R} y$ and $x<y$. Then, by Lemma 4.2, $y y^{-1} x<x x^{-1} y$ and so, by (15f), $x^{-1}\left(x^{-1}\right)^{-1} y^{-1}=x^{-1} x y^{-1}<y^{-1} y x^{-1}$ $=y^{-1}\left(y^{-1}\right)^{-1} x^{-1}$. Since $x \mathscr{R} y$, we have $x x^{-1}=y y^{-1}$ and so $x^{-1} \mathscr{L} y^{-1}$. Hence, by Lemma 4.2, we have $y^{-1}<x^{-1}$.

(15h) implies (15i). In fact, we suppose that $x y$ is positive. Then, by Lemma 4.8, $y y^{-1} x^{-1}<x^{-1} x y$. Now $y y^{-1} x^{-1} \mathscr{R} x^{-1} x y y^{-1} \mathscr{R} x^{-1} x y$ and so, by $(15 \mathrm{~h}), y^{-1} x^{-1} x$ $=\left(x^{-1} x y\right)^{-1}<\left(y y^{-1} x^{-1}\right)^{-1}=x y y^{-1}$. Therefore, by Lemma 4.1, $x x^{-1} y^{-1}<y^{-1} y x$ and so, by Lemma $4.8, y x=\left(y^{-1}\right)^{-1} x$ is positive.

(15i) implies (15j). In fact, we suppose that $x y^{-1} y$ is positive. Then, by (15i), $y x y^{-1}=y\left(x y^{-1}\right)$ is positive.

(15j) implies (15l). In fact, we suppose that $y$ is positive. Then, by Lemma 4.6, $y x x^{-1}$ is nonnegative. If $y x x^{-1}$ is positive, then, by $(15 \mathrm{j}), x^{-1} y x$ is positive. If $y x x^{-1}$ is idempotent, then

$$
\left(x^{-1} y x\right)^{2}=x^{-1} y x x^{-1} y x x^{-1} x=x^{-1}\left(y x x^{-1}\right)^{2} x=x^{-1}\left(y x x^{-1}\right) x=x^{-1} y x
$$

and so $x^{-1} y x$ is idempotent.

(15l) implies (15m). In fact, we suppose that $x^{-1} y$ is not idempotent and $x<y$. Then, by Lemma 3.2, $y y^{-1} x \neq x x^{-1} y$. By way of contradiction, we assume that $x x^{-1} y<y y^{-1} x$ were true. Then $x x^{-1} y y^{-1} y=x x^{-1} y<y y^{-1} x=x x^{-1} y y^{-1} x$, and so 
$y<x$, which is a contradiction. Thus we have $y y^{-1} x<x x^{-1} y$. Hence, by Lemma 4.8, $x^{-1} y$ is positive. Therefore, by (151), $(x z)^{-1}(y z)=z^{-1} x^{-1} y z$ is nonnegative. If $(x z)^{-1}(y z)$ is positive, then, by Lemma 4.8 ,

$$
\begin{aligned}
(x z)(x z)^{-1}(y z)(y z)^{-1}(x z) & =(y z)(y z)^{-1}(x z)<(x z)(x z)^{-1}(y z) \\
& =(x z)(x z)^{-1}(y z)(y z)^{-1}(y z)
\end{aligned}
$$

and so $x z<y z$. If $(x z)^{-1}(y z)$ is idempotent, then, by Lemma 3.2,

$$
\begin{aligned}
x y^{-1} y z & =x z z^{-1} y^{-1} y z=(x z)(y z)^{-1}(y z) \\
& =(y z)(x z)^{-1}(x z)=y z z^{-1} x^{-1} x z=y x^{-1} x z
\end{aligned}
$$

and also, by Lemma 4.1, $x y^{-1} y<y x^{-1} x$. Hence, by Lemma 4.10, $x z=y z$.

$(15 \mathrm{~m})$ implies (15k). In fact, we suppose that $y y^{-1} x$ is positive. Then, by Lemma 4.3, $x^{-1} y y^{-1} x=\left(y y^{-1} x\right)^{-1}\left(y y^{-1} x\right)<y y^{-1} x$ and also $\left(y y^{-1} x\right)\left(x^{-1} y y^{-1} x\right)^{-1}=y y^{-1} x$ is not idempotent. Hence, by Lemma 3.2, $\left(x^{-1} y y^{-1} x\right)^{-1}\left(y y^{-1} x\right)$ is not idempotent. Therefore by $(15 \mathrm{~m}),\left(y^{-1} x y\right)^{-1}\left(y^{-1} x y\right)=y^{-1}\left(\left(x^{-1} y y^{-1} x\right) y\right) \leqq y^{-1}\left(\left(y y^{-1} x\right) y\right)=y^{-1} x y$. Hence, by Lemma $4.4^{\prime}, y^{-1} x y$ is nonnegative. By way of contradiction, we assume that $y^{-1} x y$ is idempotent. Then

$$
\left(y y^{-1} x\right)^{3}=y\left(y^{-1} x y\right)^{2} y^{-1} x=y\left(y^{-1} x y\right) y^{-1} x=\left(y y^{-1} x\right)^{2}
$$

and so, by Lemma $3.1, y y^{-1} x$ is an idempotent, which is a contradiction. Hence $y^{-1} x y$ is positive.

(15k) implies (15g). In fact, we suppose that $x \mathscr{L} y$ and $x<y$. Then, by Lemma 4.9, $\left(x^{-1} y y^{-1}\right)\left(x^{-1} y y^{-1}\right)^{-1}\left(x^{-1} y\right)=x^{-1} y$ is positive. Therefore, by (15k),

$$
\left(x^{-1} y y^{-1}\right)^{-1}\left(x^{-1} y\right)\left(x^{-1} y y^{-1}\right)=x x^{-1} y x^{-1} y y^{-1}
$$

is positive. Hence, by Lemma $4.8,\left(y^{-1}\right)^{-1} x^{-1}=y x^{-1}$ is positive. Since $x \mathscr{L} y$, we have $x^{-1} \mathscr{R} y^{-1}$. Hence, by Lemma $4.9, y^{-1}<x^{-1}$.

(15g) implies (15d). In fact, we suppose that $x \mathscr{L} y$ and $y x^{-1}$ is positive. By way of contradiction, we assume that $x<y$ is false. If $y<x$, then, by $(15 \mathrm{~g}), x^{-1}<y^{-1}$. Moreover, since $x \mathscr{L} y$, we have $x^{-1} \mathscr{R} y^{-1}$. Hence, by Lemma $4.9, x y^{-1}=\left(x^{-1}\right)^{-1} y^{-1}$ is positive and so, by Lemma $4.5, y x^{-1}=\left(x y^{-1}\right)^{-1}$ is negative, which is a contradiction. If $y=x$, then $y x^{-1}=x x^{-1}$ is an idempotent, which is also a contradiction. Thus we have $x<y$.

(15d) implies (15b). In fact. we suppose that $x \mathscr{L} y$ and $x<y$. By way of contradiction, we assume that $y x^{-1}$ is not positive. If $y x^{-1}$ is negative, then, by Lemma 4.5, $x y^{-1}=\left(y x^{-1}\right)^{-1}$ is positive, and so, by (15d), $y<x$, which is a contradiction. If $y x^{-1}$ is an idempotent, then, by Lemma 3.2, $y y^{-1} x=x x^{-1} y$. Hence, by Lemma 4.2, $x=y$, which is a contradiction. Thus $y x^{-1}$ is positive.

(15b) implies $\left(15^{\prime}\right)$. In fact, we suppose that $x x^{-1}=y^{-1} y=z^{-1} z$ and $y \leqq z$. First we suppose $y<z$. Now $y \mathscr{L} y^{-1} y=z^{-1} z \mathscr{L} z$ and so, by $(15 b),(z x)(y x)^{-1}=z x x^{-1} y^{-1}$ $=z y^{-1} y y^{-1}=z y^{-1}$ is positive. Hence, by Lemma $4.5,(y x)(z x)^{-1}=\left((z x)(y x)^{-1}\right)^{-1}$ 
is negative. Moreover, since $y \mathscr{L} z$, we have $y x \mathscr{L} z x$. Hence, by (15b), $z x<y x$ does not hold and so $y x \leqq z x$. In the case when $y=z$, we have $y x=z x$.

This completes the proof of Theorem 5.6.

THEOREM 5.7. Let $S$ be a left ordered inverse semigroup in which, for every pair of positive elements $x, y$ of $S$, there exists a natural number $n$ such that $x \leqq y^{n}$. Then $S$ satisfies the condition $\left(15^{\prime}\right)$.

Proof. We prove $S$ satisfies the condition (15l). To do this, suppose that $v$ is positive. Then, by Lemma $4.6, v u u^{-1}$ is nonnegative.

(i) The case when both $u$ and $v u u^{-1}$ are positive. By assumption, there exists a natural number $n$ such that $u \leqq\left(v u u^{-1}\right)^{n}$. Without loss of generality, we assume that $n$ is the least natural number such that $u \leqq\left(v u u^{-1}\right)^{n}$. By way of contradiction, we assume that $u^{-1} v u$ is negative. Then, by Lemma $4.5, u^{-1} v^{-1} u=\left(u^{-1} v u\right)^{-1}$ is positive. Hence, by Lemma $4.3, u^{-1} v^{-1} u u^{-1} v u=\left(u^{-1} v^{-1} u\right)\left(u^{-1} v^{-1} u\right)^{-1}<u^{-1} v^{-1} u$ $=u^{-1} v^{-1} u u^{-1} u$. Therefore $\left(v u u^{-1}\right) u=v u<u \leqq\left(v u u^{-1}\right)^{n}$. If $n>1$, then we have $u<\left(v u u^{-1}\right)^{n-1}$, which contradicts the minimality of $n$. Next we suppose that $n=1$. Then $v u<v u u^{-1}$ and so $u<u u^{-1}$. Hence, by Lemma $4.4, u$ is negative, which contradicts the assumption. Thus $u^{-1} v u$ is nonnegative.

(ii) The case when $v u u^{-1}$ is idempotent. We have $\left(u^{-1} v u\right)^{3}=u^{-1}\left(v u u^{-1}\right)^{2} v u$ $=u^{-1}\left(v u u^{-1}\right) v u=\left(u^{-1} v u\right)^{2}$. Hence, by Lemma 3.1, $u^{-1} v u$ is idempotent.

(iii) The case when $u$ is idempotent. By Lemma $4.6, u^{-1} v u=u v u$ is nonnegative.

(iv) The case when $u$ is negative. By way of contradiction, we assume that $u^{-1} v u$ were negative. Then, by Lemma $4.5, u^{-1} v^{-1} u=\left(u^{-1} v u\right)^{-1}$ is positive. By assumption, $u$ is negative and so, by Lemma $4.5, u^{-1}$ is positive. Hence, by (i) and (ii) proved above, $\left(u u^{-1}\right) v^{-1}\left(u u^{-1}\right)=\left(u^{-1}\right)^{-1}\left(u^{-1} v^{-1} u\right) u^{-1}$ is nonnegative. On the other hand, by Lemma $4.5, v^{-1}$ is negative and so, by Lemma $4.7,\left(u u^{-1}\right) v^{-1}\left(u u^{-1}\right)$ is nonpositive. Hence $u u^{-1} v^{-1} u u^{-1}$ is an idempotent. Therefore

$$
u^{-1} v u=u^{-1}\left(u u^{-1} v^{-1} u u^{-1}\right)^{-1} u=u^{-1}\left(u u^{-1} v^{-1} u u^{-1}\right) u
$$

is an idempotent, which contradicts the assumption. Hence $u^{-1} v u$ is nonnegative.

Thus we have proved that $S$ satisfies (15l). Hence, by Theorem 5.6, $S$ satisfies $\left(15^{\prime}\right)$.

THEOREM 5.8. Let $S$ be a left ordered inverse semigroup and let $E$ be the set of all idempotents of $S$. Then each one of the following conditions is equivalent to the condition $\left(16^{\prime}\right)$ in Theorem 5.4:

(16a) if $x^{-1} y \in E$ and $x \leqq y$, then $x x^{-1} \leqq y y^{-1}$;

(16b) if $x^{-1} y \in E$ and $x \leqq y$, then $x^{-1} \leqq y^{-1}$;

(16c) if $f, g \in E$ and $f \leqq g$, then $f z \leqq g z$ for every $z \in S$;

(16d) if $x^{-1} y \in E$ and $x \leqq y$, then $x z \leqq y z$ for every $z \in S$.

Proof. (16') implies (16a). In fact, we suppose that $x^{-1} y \in E$ and $x \leqq y$. Then, by Lemma 3.2, $y y^{-1} x=x x^{-1} y$ and so, by the condition (14') in Theorem 3.4, we 
have $x^{-1} x \leqq y^{-1} y$. Hence, by Lemma $1.1, x^{-1} x \leqq\left(x^{-1} x\right)\left(y^{-1} y\right) \leqq y^{-1} y$. Therefore, by $\left(16^{\prime}\right)$,

$$
\begin{aligned}
x x^{-1} & =x\left(x^{-1} x\right) x^{-1} \leqq x\left(x^{-1} x y^{-1} y\right) x^{-1}=x y^{-1} y x^{-1}, \\
y x^{-1} x y^{-1} & =y\left(x^{-1} x y^{-1} y\right) y^{-1} \leqq y\left(y^{-1} y\right) y^{-1}=y y^{-1}
\end{aligned}
$$

By assumption, $x^{-1} y \in E$ and so, by Lemma 3.2, $x y^{-1} y x^{-1}=y x^{-1}=x x^{-1} y y^{-1}$ $=x y^{-1}=y x^{-1} x y^{-1}$. Hence $x x^{-1} \leqq x y^{-1} y x^{-1}=y x^{-1} x y^{-1} \leqq y y^{-1}$.

(16a) implies (16b). In fact, we suppose that $x^{-1} y \in E$ and $x \leqq y$. Then, by (16a), $x^{-1}=x^{-1} x x^{-1} \leqq x^{-1} y y^{-1}, y^{-1} x x^{-1} \leqq y^{-1} y y^{-1}=y^{-1}$. Since $x^{-1} y \in E$, we have, by Lemma 3.2, $x^{-1} y y^{-1}=\left(y y^{-1} x\right)^{-1}=\left(x x^{-1} y\right)^{-1}=y^{-1} x x^{-1}$. Hence $x^{-1} \leqq x^{-1} y y^{-1}$ $=y^{-1} x x^{-1} \leqq y^{-1}$.

(16b) implies (16d). In fact, we suppose that $x^{-1} y \in E$ and $x \leqq y$. Then, by (16b), we have $x^{-1} \leqq y^{-1}$ and so $z^{-1} x^{-1} \leqq z^{-1} y^{-1}$. Now, by assumption, $x^{-1} y \in E$ and so, by Lemma 3.2, $x y^{-1} \in E$. Hence

$$
\left(z^{-1} x^{-1}\right)^{-1}\left(z^{-1} y^{-1}\right)=x z z^{-1} y^{-1}=\left(x z z^{-1} x^{-1}\right)\left(x y^{-1}\right) \in E .
$$

Therefore, by (16b), $x z=\left(z^{-1} x^{-1}\right)^{-1} \leqq\left(z^{-1} y^{-1}\right)^{-1}=y z$.

(16d) implies (16c). In fact, replacing $x$ and $y$ in (16d) by $f$ and $g$ respectively, we obtain (16c).

(16c) implies (16'). In fact we suppose that $e, f, g \in E, x \in R_{e}, f \leqq e, g \leqq e$ and $f \leqq g$. Then, by (16c), $f x \leqq g x$ and so $x^{-1} f x \leqq x^{-1} g x$.

REMARK. The equivalences of $\left(15^{\prime}\right)$ and $(15 \mathrm{~m})$ in Theorem 5.6 and of $\left(16^{\prime}\right)$ and (16d) in Theorem 5.8 give an alternative proof of Theorem 5.4.

6. The left orderability and the orderability of inverse semigroups. A semigroup $S$ is called left orderable if $S$ admits an order to make $S$ a left ordered semigroup. $S$ is called orderable if $S$ admits an order to make $S$ an ordered semigroup. In Theorem 6.3 we give a condition in order that an inverse semigroup $S$ is left orderable and in Theorem 6.8 we give a condition in order that $S$ is orderable.

THEOREM 6.1. A commutative idempotent semigroup $S$ is orderable if and only if the semilattice $S$ induced by the natural partial ordering is a tree semilattice, in which the branch number at every element is at most two.

Proof. The 'only if' part is given by Lemma 1.3.

We prove the 'if' part. Suppose that $S$ is a commutative idempotent semigroup such that the semilattice $(S, \preceq)$ is a tree semilattice in which the branch number at every element is at most two. When $a$ is a branching element, then, by assumption, there are exactly two branches at $a$. In this case, we denominate an arbitrary one of the branches as the former branch and the other as the latter branch. When $a$ is an intermediate element, then there is exactly one branch at $a$. In this case, we denominate the branch as either one of the former branch or the latter branch. When $a$ is a maximal element, there is no branch at $a$ to denominate it. Now we define an order $\leqq$ in $S$ by: $e \leqq f$ if and only if either 
(a) $e f<e, e f \prec f$, $e$ lies in the former branch at $e f$ and $f$ lies in the latter branch at $e f$, or

(b) $e f=e \prec f$ and $f$ lies in the latter branch at $e f$, or

(c) $e f=f \prec e$ and $e$ lies in the former branch at $e f$, or

(d) $e=f$.

First we show that the relation $\leqq$ is really an order in $S$. It is almost trivial that the relation is reflexive and antisymmetric. Now we suppose that $e \leqq f$ and $f \leqq g$. We have $e f \leqq f$ and $f g \leqq f$ and, since $(S, \preceq)$ is a tree semilattice, $e f$ and $f g$ are comparable in $(S, \supseteqq)$.

(i) The case when $e f \prec f g$. We have $e f=e f g \prec f g$. Since $e g \leqq g$ and $f g \leqq g$, eg and $f g$ are comparable in $(S, \supseteqq)$. But, since $e f g \neq f g$, we have $e g \prec f g$ and so $e g=e f g$ $=e f \prec f g \leqq f$. By assumption, $e \leqq f$ and so $f$ lies in the latter branch at $e f=e g$ and also either $e=e f=e g$ or $e$ lies in the former branch at $e f=e g$. Moreover, since $e g \prec f g, f$ and $g$ lie in the same branch at $e g$ and so $g$ lies in the latter branch at $e g$. Hence we have $e \leqq g$.

(ii) The case when $f g \prec e f$. We can prove $e \leqq g$ in a similar way to (i).

(iii) The case when $e f=f g$. We have $e f=f g=e f g$. By way of contradiction, we assume that $e f g \neq e g$. Then, since $e \leqq f$ and $e f=e f g \prec e g \leqq e, e$ lies in the former branch at $e f g$. Since $f \leqq g$ and $f g=e f g \prec e g \leqq g, g$ lies in the latter branch at $e f g$. But, since $e f g \prec e g, e$ and $g$ lie in the same branch at $e f g$, which is a contradiction. Hence we have $e f=f g=e f g=e g$. Since $e \leqq f$, either $e=e f=e g$ or $e$ lies in the former branch at $e f=e g$. Also, since $f \leqq g$, either $g=f g=e g$ or $g$ lies in the latter branch at $f g=e g$. Hence, in all cases, we have $e \leqq g$.

This proves the relation $\leqq$ is transitive. Now we take arbitrary elements $e, f$ of $S$. If $e f \prec e$ and $e f \prec f$, $e$ and $f$ lie in different branches at $e f$. When $e$ lies in the former and $f$ lies in the latter branch at $e f$, then $e \leqq f$, while when $e$ lies in the latter and $f$ lies in the former branch at $e f$, then $f \leqq e$. Next we suppose that at least one of $e$ and $f$, say $e$, is equal to $e f$. When $f$ lies in the former branch at $e f$, then $f \leqq e$, while when $f$ lies in the latter branch at $e f$, then $e \leqq f$, and finally when $f=e f$, then $e=f$. Hence the relation $\leqq$ is a simple order.

Finally we prove that the order $\leqq$ is compatible with the semigroup operation. Suppose that $e \leqq f$. Since $e g \leqq g$ and $f g \leqq g$, $e g$ and $f g$ are comparable in $(S, \leqq)$. First we consider the case when $e g \prec f g$. We have $e g=e f g \prec f g$. Also, since $e f \leqq f$, $f g \leqq f$, $e f$ and $f g$ are comparable in $(S, \preceq)$. But, since $e f g \neq f g$, we have $e f \prec f g$ and so $e f=e f g \prec f g$. Since $e f \prec f g \leqq f$ and $e \leqq f, f$ lies in the latter branch at $e f$. Since $e f \prec f g=f(f g), f g$ and $f$ lie in the same branch at $e f$ and so $f g$ lies in the latter branch at $e f=e f g=(e g)(f g)$. Also we have $e g=e f g=(e g)(f g)$ and so $e g \leqq f g$. In the case when $f g \prec e g$, we can prove $e g \leqq f g$ in a similar way. Finally in the case when $e g=f g$, there is nothing to prove.

This completes the proof of Theorem 6.1.

For a subset $X$ of an inverse semigroup $S$ we denote the set $\left\{x^{-1} ; x \in X\right\}$ by $X^{-1}$. 
THEOREM 6.2. Let $S$ be an inverse semigroup which contains no elements of finite order except idempotents. If the set $E$ of all idempotents of $S$ is an ordered commutative idempotent semigroup and if $S$ contains a subsemigroup $P$ such that $P \cap P^{-1}$ $=E$ and $P \cup P^{-1}=S$, then we can define such an order in $S$ in one and only one way that $S$ is a left ordered inverse semigroup, $P$ is the nonnegative part of the left ordered inverse semigroup $S$ and, on the set $E$, the order in $S$ coincides with the given order in $E$.

Proof. First we prove the 'in one way' part. For each $e \in E$, we define an order $\leqq$ in $R_{e}$ by for $x, y \in R_{e}, x \leqq y$ if and only if $x^{-1} y \in P$.

First we prove that the relation $\leqq$ in $R_{e}$ is really a simple order. Since $x^{-1} x \in E \subseteq P$, the relation $\leqq$ is reflexive. Next we suppose that $x \leqq y$ and $y \leqq x$. Then $x^{-1} y \in P$ and $\left(x^{-1} y\right)^{-1}=y^{-1} x \in P$. Hence $x^{-1} y \in P \cap P^{-1}=E$. By assumption, $S$ contains no elements of finite order except idempotents and so, by Lemma 3.2, $y y^{-1} x$ $=x x^{-1} y$. Moreover $x, y \in R_{e}$ and so, by Lemma 4.2, we have $x=y$. Hence the relation $\leqq$ is antisymmetric. Now we suppose that $x \leqq y$ and $y \leqq z$. Then $x^{-1} y \in P$ and $y^{-1} z \in P$ and so

$$
x^{-1} z=x^{-1} x x^{-1} z=x^{-1} e z=x^{-1} y y^{-1} z=\left(x^{-1} y\right)\left(y^{-1} z\right) \in P .
$$

Hence the relation $\leqq$ is transitive. Finally we take $x, y \in R_{e}$ arbitrarily. Since $P \cup P^{-1}=S$, we have either $x^{-1} y \in P$ or $x^{-1} y \in P^{-1}$. If $x^{-1} y \in P$, then $x \leqq y$. If $x^{-1} y \in P^{-1}$, then $y^{-1} x=\left(x^{-1} y\right)^{-1} \in P$ and so $y \leqq x$. Hence the relation $\leqq$ is a simple order.

Next we prove that $S$ satisfies the conditions $\left(11^{\prime}\right),\left(12^{\prime}\right)$ and $\left(13^{\prime}\right)$ in Theorem 3.4. By assumption, $\left(11^{\prime}\right)$ is satisfied. Now we suppose that $e, f \in E, x, y \in R_{e}, x \leqq y$ in $R_{e}$ and $f \leqq e$. Then $f x, f y \in R_{f}$ and $(f x)^{-1}(f y)=x^{-1} f y=x^{-1} f x x^{-1} y$. But $x^{-1} f x \in E$ $\subseteq P$ and, since $x, y \in R_{e}$ and $x \leqq y$, we have $x^{-1} y \in P$. Hence $(f x)^{-1}(f y)$ $=x^{-1} f x x^{-1} y \in P$ and so $f x \leqq f y$ in $R_{f}$. Thus $\left(12^{\prime}\right)$ is satisfied. Finally we suppose that $e, f \in E, x \in R_{e}, x^{-1} x=f, y, z \in R_{f}$ and $y \leqq z$ in $R_{f}$. Then $x y, x z \in R_{e}$ and

$$
(x y)^{-1}(x z)=y^{-1} x^{-1} x z=y^{-1} f z=y^{-1} y y^{-1} z=y^{-1} z \in P .
$$

Hence $x y \leqq x z$ in $R_{e}$ and so $\left(13^{\prime}\right)$ is satisfied.

Therefore, by Theorem 3.6, when we define an order in $S$ by $\left(14^{\prime}\right)$ in Theorem 3.4, $S$ is a left ordered inverse semigroup and, on the set $E$, the order of $S$ coincides with the given order in $E$ and also, for each $e \in E$, on the set $R_{e}$, the order of $S$ coincides with the order in $R_{e}$ constructed above. Now we prove that $P$ is the nonnegative part of the left ordered inverse semigroup $S$. Evidently $x \in P$ if and only if $\left(x x^{-1}\right)^{-1} x \in P$. Since $x \mathscr{R} x x^{-1}, x \in P$ if and only if $x x^{-1} \leqq x$ with respect to the order in $R_{x x}{ }^{-1}$, and so, if and only if $x x^{-1} \leqq x$ with respect to the order in $S$. Hence, by Lemma $4.4^{\prime}, x \in P$ if and only if $x$ is nonnegative. Therefore $P$ is the nonnegative part of the left ordered inverse semigroup $S$. This completes the proof of the 'in one way' part. 
Next we prove the 'in only one way' part. We denote by $(S, \leqq)$ the left ordered inverse semigroup constructed above. Moreover we suppose that $\left(S, \leqq_{1}\right)$ is an arbitrary left ordered inverse semigroup such that $P$ is the nonnegative part of $\left(S, \leqq_{1}\right)$ and, on the set $E$, the order $\leqq_{1}$ coincides with the given order in $E$. Then, on the set $E$, the orders $\leqq$ and $\leqq_{1}$ coincide with each other. Let $e \in E$ and let $x, y \in R_{e}$. Then,by Lemma 4.9, $x<_{1} y$ if and only if $x^{-1} y$ is positive in $\left(S, \leqq_{1}\right)$. Also, by Lemma 4.2, $x=y$ if and only if $y y^{-1} x=x x^{-1} y$ and so, by Lemma 3.2, if and only if $x^{-1} y$ is idempotent. Hence $x \leqq_{1} y$ if and only if $x^{-1} y$ is nonnegative in $\left(S, \leqq_{1}\right)$ and so if and only if $x^{-1} y \in P$. Thus the orders $\leqq$ and $\leqq_{1}$ coincide with each other on $R_{e}$ for each $e \in E$. By Theorem $3.4,\left(S, \leqq_{1}\right)$ satisfies the conditions $\left(11^{\prime}\right),\left(12^{\prime}\right)$ and $\left(13^{\prime}\right)$. Hence, by Theorem 3.6 , the orders $\leqq$ and $\varliminf_{1}$ coincide with each other on the set $S$. This completes the proof of the 'in only one way' part.

THEOREM 6.3. Let $S$ be an inverse semigroup and let $E$ be the set of all idempotents of $S$. Then $S$ is left orderable if and only if it satisfies the following three conditions:

(A) $S$ contains no elements of finite order except idempotents;

(B) $S$ contains a subsemigroup $P$ such that $P \cap P^{-1}=E$ and $P \cup P^{-1}=S$;

(C) the semilattice $E$ is a tree semilattice, in which the branch number at every element is at most two.

Proof. First we prove the 'only if' part and suppose that $S$ is a left ordered inverse semigroup. By Lemma 3.1, $S$ satisfies (A). By Lemma 4.6, the nonnegative part $P$ of $S$ is a subsemigroup. By Lemma $4.5, P^{-1}$ is the nonpositive part of $S$ and so $S$ satisfies (B). By Theorem 6.1, $S$ satisfies (C).

Next we prove the 'if' part and suppose that $S$ is an inverse semigroup satisfying the conditions (A), (B) and (C). By Theorem 6.1, E can be considered as an ordered commutative idempotent semigroup and so, by Theorem $6.2, S$ can be considered as a left ordered inverse semigroup.

Let $S$ be an inverse semigroup in which there is no element of finite order except idempotents and let the semilattice $E$ constituted by all idempotents of $S$ form a tree semilattice. We denote the set of all branches in $E$ by $\mathfrak{B}$. Let $B, B^{\prime} \in \mathfrak{B}$ and we suppose that $B$ is a branch at $e$ and $B^{\prime}$ is a branch at $f$. Then $B$ is said to be transferred to $B^{\prime}$ by a translation, if there exist $x, y \in S$ such that $x x^{-1}=e, x^{-1} x=f$, $y y^{-1} \in B, y^{-1} y \in B^{\prime}, x^{-1} y \in E$.

LEMMA 6.4. The relation that a branch $B$ is transferred to a branch $B^{\prime}$ by a translation is an equivalence relation on $\mathfrak{B}$.

Proof. (i) Reflexivity. We suppose that $B \in \mathfrak{B}$ is a branch at $e$. We take $g \in B$ arbitrarily. Then $e e^{-1}=e=e^{-1} e, g g^{-1}=g=g^{-1} g \in B, e^{-1} g=e g \in E$. Hence, by definition, $B$ is transferred to $B$ by a translation.

(ii) Symmetry. We suppose that a branch $B$ at $e$ is transferred to a branch $B^{\prime}$ at $f$ by a translation. Then there exist $x, y \in S$ such that $x x^{-1}=e, x^{-1} x=f, y y^{-1} \in B$, 
$y^{-1} y \in B^{\prime}, x^{-1} y \in E$. By Lemma $3.2,\left(x^{-1}\right)^{-1} y^{-1}=x y^{-1} \in E$ and so $B^{\prime}$ is transferred to $B$ by a translation.

(iii) Transitivity. We suppose that a branch $B$ at $e$ is transferred to a branch $B^{\prime}$ at $f$ by a translation and $B^{\prime}$ is transferred to a branch $B^{\prime \prime}$ at $g$ by a translation. Then there exist $x, y, u, v \in S$ such that

$$
\begin{aligned}
& x x^{-1}=e, \quad x^{-1} x=f, \quad y y^{-1} \in B, \quad y^{-1} y \in B^{\prime}, \quad x^{-1} y \in E ; \\
& u u^{-1}=f, \quad u^{-1} u=g, \quad v v^{-1} \in B^{\prime}, \quad v^{-1} v \in B^{\prime \prime}, \quad u^{-1} v \in E .
\end{aligned}
$$

Now we have

$$
\begin{aligned}
& (x u)(x u)^{-1}=x u u^{-1} x^{-1}=x f x^{-1}=x x^{-1} x x^{-1}=x x^{-1}=e, \\
& (x u)^{-1}(x u)=u^{-1} x^{-1} x u=u^{-1} f u=u^{-1} u u^{-1} u=u^{-1} u=g .
\end{aligned}
$$

Since $x x^{-1}=e \prec y y^{-1}$ and $y v v^{-1} y^{-1} \leqq y y^{-1}, x x^{-1}$ and $y v v^{-1} y^{-1}$ are comparable in the semilattice $(E, \leqq)$. By way of contradiction, we assume that $y v v^{-1} y^{-1} \leqq x x^{-1}$ is true. Then $y v v^{-1} y^{-1} x x^{-1}=y v v^{-1} y^{-1}$ and so $v v^{-1} y^{-1} x x^{-1} y=$ $y^{-1}\left(y v v^{-1} y^{-1} x x^{-1}\right) y=y^{-1}\left(y v v^{-1} y^{-1}\right) y=y^{-1} y v v^{-1}$. Now $x^{-1} y \in E$ and so, by Lemma 3.2, $\quad x x^{-1} y=y y^{-1} x=y y^{-1} x x^{-1} x=x x^{-1} x=x$. Hence $y^{-1} y v v^{-1}$ $=v v^{-1} y^{-1} x x^{-1} y=v v^{-1}\left(x x^{-1} y\right)^{-1}\left(x x^{-1} y\right)=v v^{-1} x^{-1} x=v v^{-1} f=f$. On the other hand, $y^{-1} y$ and $v v^{-1}$ lie in the same branch $B^{\prime}$ at $f$ and so $f \prec y^{-1} y v v^{-1}$, which is a contradiction. Hence

$$
e=x x^{-1} \prec y v v^{-1} y^{-1}=\left(y y^{-1}\right)\left(y v v^{-1} y^{-1}\right) .
$$

Therefore $y y^{-1}$ and $y v v^{-1} y^{-1}$ lie in the same branch at $e$ and so $(y v)(y v)^{-1}$ $=y v v^{-1} y^{-1} \in B$. Similarly we can prove that $(y v)^{-1}(y v) \in B^{\prime \prime}$. Moreover, since $x^{-1} y, u^{-1} v \in E$, we have

$$
(x u)^{-1}(y v)=u^{-1} x^{-1} y v=\left(u^{-1}\left(x^{-1} y\right) u\right)\left(u^{-1} v\right) \in E .
$$

Hence, by definition, $B$ is transferred to $B^{\prime \prime}$ by a translation.

Let $B, B^{\prime} \in \mathfrak{B}$ and we suppose that $B$ is a branch at $e$ and $B^{\prime}$ is a branch at $f$. Then $B$ is said to be transferred to $B^{\prime}$ by a conversion if $e=f$ and $B$ and $B^{\prime}$ are different branches at $e$. A branch $B$ is said to be connected with a branch $B^{\prime}$ if there exist a finite number of branches $B=B_{1}, B_{2}, \ldots, B_{n}=B^{\prime}(n \geqq 2)$ such that $B_{i}$ is transferred to $B_{i+1}$ by either a translation or a conversion for every $1 \leqq i \leqq n-1$.

LEMMA 6.5. The relation that a branch $B$ is connected with a branch $B^{\prime}$ is an equivalence relation on $\mathfrak{B}$.

Proof. (i) Reflexivity. By Lemma 6.4, a branch $B$ is transferred to $B$ by a translation and so $B$ is connected with $B$.

(ii) Symmetry. We suppose that a branch $B$ is connected with a branch $B^{\prime}$. Then there exist a finite number of branches $B=B_{1}, B_{2}, \ldots, B_{n}=B^{\prime}$ such that $B_{i}$ is transferred to $B_{i+1}$ by either a translation or a conversion for every $1 \leqq i \leqq n-1$. If $B_{i}$ is transferred to $B_{i+1}$ by a translation, then, by Lemma $6.4, B_{i+1}$ is transferred 
to $B_{i}$ by a translation. If $B_{i}$ is transferred to $B_{i+1}$ by a conversion, it is evident from the definition, that $B_{i+1}$ is transferred to $B_{i}$ by a conversion. Hence, in both cases, $B^{\prime}$ is connected with $B$.

(iii) The transitivity of the relation of connectedness is almost trivial.

THEOREM 6.6. Let $S$ be an inverse semigroup which contains no elements of finite order except idempotents and let $E$ be the set of all idempotents of $S$. Then we can define an order in $E$ to make $E$ an ordered commutative idempotent semigroup satisfying the condition $\left(16^{\prime}\right)$ in Theorem 5.4 if and only if $S$ satisfies the condition:

$\left(C^{*}\right)$ The semilattice $E$ is a tree semilattice and, when a branch $B$ is connected with a branch $B^{\prime}$ and we choose branches $B=B_{1}, B_{2} \ldots, B_{n}=B^{\prime}$ such that $B_{i}$ is transferred to $B_{i+1}$ by either a translation or a conversion for every $1 \leqq i \leqq n-1$, whether the number of conversions included in the transfers is even or odd is determined by $B$ and $B^{\prime}$, irrespective of the choice of branches $B_{i}(1 \leqq i \leqq n)$.

Proof. First we prove the 'only if' part and suppose that $E$ is an ordered commutative idempotent semigroup satisfying the condition $\left(16^{\prime}\right)$. By Theorem 6.1, $E$ is a tree semilattice.

$1^{\circ}$. Let $B$ be a branch at $e$ and let $g \in B$.

(a) If $g<e$, then $f<e$ for every $f \in B$;

(b) if $g>e$, then $f>e$ for every $f \in B$.

In fact, we suppose that $g<e$. By way of contradiction, we assume that $f \geqq e$ for some $f \in B$. Then, by Lemma $1.2, g f \leqq e$. On the other hand, $f$ and $g$ lie in the same branch $B$ at $e$. Hence $e \prec g f$, which is a contradiction. Thus we have (a). (b) can be proved in a similar way.

$2^{\circ}$. Let $B$ and $B^{\prime}$ be different branches at $e$ and let $f \in B$ and $g \in B^{\prime}$.

(a) If $f<e$, then $g>e$;

(b) if $f>e$, then $g<e$.

In fact, by way of contradiction, we assume that $f<e$ and $g \leqq e$. Since $g$ lies in a branch $B^{\prime}$ at $e$, we have $g \neq e$. Hence $f<e$ and $g<e$, and so, by Lemma $1.1, f g<e$. On the other hand, $f$ and $g$ lie in different branches at $e$ and so $f g=e$, which is a contradiction. Thus we have (a). (b) can be proved in a similar way.

Let $B$ be a branch at $e$ such that $f<e$ for some $f \in B$. By $2^{\circ}, B$ is the only branch at $e$ carrying this property, which is called the former branch at $e$. Let $B^{\prime}$ be a branch at $e$ such that $f>e$ for some $f \in B^{\prime}$. Then $B^{\prime}$ is the only branch at $e$ carrying this property, which is called the latter branch at $e$. By $1^{\circ}$, a branch at $e$ cannot be the former branch and the latter branch at $e$ at the same time.

$3^{\circ}$. Suppose that a branch $B$ at $e$ is transferred to a branch $B^{\prime}$ at $f$ by a translation.

(a) If $B$ is the former branch at e, then $B^{\prime}$ is the former branch at $f$;

(b) if $B$ is the latter branch at $e$, then $B^{\prime}$ is the latter branch at $f$.

In fact, by assumption, there exist $x, y \in S$ such that

$$
x x^{-1}=e, \quad x^{-1} x=f, \quad y y^{-1} \in B, \quad y^{-1} y \in B^{\prime}, \quad x^{-1} y \in E .
$$


First we suppose that $B$ is the former branch at $e$. Then $y y^{-1}<e=x x^{-1}$. Moreover, since $y y^{-1} \in B$ and $B$ is a branch at $e$, we have $x x^{-1}=e \prec y y^{-1}$. Hence, by $\left(16^{\prime}\right)$, $y^{-1} y=y^{-1}\left(y y^{-1}\right) y \leqq y^{-1} x x^{-1} y$. But, if $y^{-1} y=y^{-1} x x^{-1} y$ were true, then $y y^{-1}$ $=y\left(y^{-1} y\right) y^{-1}=y\left(y^{-1} x x^{-1} y\right) y^{-1}=\left(y y^{-1}\right)\left(x x^{-1}\right)\left(y y^{-1}\right)=x x^{-1}$, which is a contradiction. Hence $y^{-1} y<y^{-1} x x^{-1} y$. Since $x^{-1} y \in E$, we have, by Lemma 3.2, $y^{-1} y<y^{-1} x x^{-1} y=y^{-1} x=x^{-1} x y^{-1} y$. By way of contradiction, we assume that $x^{-1} x \leqq y^{-1} y$. Then, by Lemma $1.1, x^{-1} x \leqq x^{-1} x y^{-1} y \leqq y^{-1} y$, which is a contradiction. Hence $y^{-1} y<x^{-1} x=f$ and so $B^{\prime}$ is the former branch at $f$. This proves (a). The assertion (b) can be proved in a similar way.

$4^{\circ}$. Suppose that a branch $B$ at $e$ is transferred to a branch $B^{\prime}$ at e by a conversion.

(a) If $B$ is the former branch at $e$, then $B^{\prime}$ is the latter branch at $e$;

(b) if $B$ is the latter branch at $e$, then $B^{\prime}$ is the former branch at $e$.

In fact, both (a) and (b) are immediate consequences of $2^{\circ}$.

$5^{\circ}$. S satisfies the condition $\left(\mathrm{C}^{*}\right)$.

In fact, we suppose that $B_{i}(1 \leqq i \leqq m)$ and $B_{j}^{\prime}(1 \leqq j \leqq n)$ are branches such that $B=B_{1}=B_{1}^{\prime}, B^{\prime}=B_{m}=B_{n}^{\prime}, B_{i}$ is transferred to $B_{i+1}$ by either a translation or a conversion for every $1 \leqq i \leqq m-1$ and $B_{j}^{\prime}$ is transferred to $B_{j+1}^{\prime}$ by either a translation or a conversion for every $1 \leqq j \leqq n-1$. By way of contradiction, we assume that the transfers of $B$ to $B^{\prime}$ by $B_{i}(1 \leqq i \leqq m)$ contain an even number of conversions and the transfers of $B$ to $B^{\prime}$ by $B_{j}^{\prime}(1 \leqq j \leqq n)$ contain an odd number of conversions. Then, by $3^{\circ}$ and $4^{\circ}, B^{\prime}$ is the former branch and the latter branch at the same time, which is a contradiction. Hence $S$ satisfies the condition $\left(C^{*}\right)$.

Next we prove the 'if' part and suppose that $S$ satisfies $\left(\mathrm{C}^{*}\right)$. Then, by Lemma 6.5 , the relation of connectedness is an equivalence relation on the set $\mathfrak{B}$ of all branches in the semilattice $E$. We denote the set of all equivalence classes by $\left\{\mathfrak{B}_{\lambda} ; \lambda \in \Lambda\right\}$. From each equivalence class $\mathfrak{B}_{\lambda}$, we choose one representative element $B_{\lambda} \in \mathfrak{B}_{\lambda}$ and we denominate $B_{\lambda}$ arbitrarily as either one of the former branch or the latter branch. Now we take an arbitrary branch $B \in \mathfrak{B}$ and we suppose that the equivalence class which contains $B$ is $\mathfrak{B}_{\lambda}$. Then the representative element $B_{\lambda}$ of $\mathfrak{B}_{\lambda}$ is connected with $B$ and so there exist branches $B_{\lambda}=B_{1}, B_{2}, \ldots, B_{n}=B$ such that $B_{i}$ is transferred to $B_{i+1}$ by either a translation or a conversion. If the number of conversions contained in the transfers is even, we denominate $B$ as the former or the latter branch according as $B_{\lambda}$ is the former or the latter branch. If the number of conversions contained in the transfers is odd, we denominate $B$ as the latter or the former branch according as $B_{\lambda}$ is the former or the latter branch. By the condition $\left(C^{*}\right)$, whether $B$ is the former or the latter branch is determined by $B$, irrespective of the choice of branches $B_{i}(1 \leqq i \leqq n)$. Let $B$ and $B^{\prime}$ be different branches at the same element. Then $B$ and $B^{\prime}$ belong to the same equivalence class, say $\mathfrak{B}_{\lambda}$. We suppose that the representative element $B_{\lambda}$ of $\mathfrak{B}_{\lambda}$ is transferred to $B$ by $B_{\lambda}$ $=B_{1}, B_{2}, \ldots, B_{n}=B$. Then $B_{\lambda}$ is transferred to $B^{\prime}$ by $B_{\lambda}=B_{1}, B_{2}, \ldots, B_{n}=B, B_{n+1}$ $=B^{\prime}$ and the number of conversions contained in the transfers of $B_{\lambda}$ to $B^{\prime}$ is even or odd, according as the number of conversions contained in the transfers of $B_{\lambda}$ to $B$ 
is odd or even. Hence $B^{\prime}$ is the former branch or the latter branch according as $B$ is the latter branch or the former branch. Now we define an order in $E$ by: $e \leqq f$ if and only if either

(a) $e f \prec e, e f \prec f$, $e$ lies in the former branch at $e f$ and $f$ lies in the latter branch at $e f$, or

(b) $e f=e \prec f$ and $f$ lies in the latter branch at $e f$, or

(c) $e f=f \prec e$ and $e$ lies in the former branch at $e f$, or

(d) $e=f$.

In the same way as in the proof of Theorem 6.1 , we can prove that the relation $\leqq$ is really a simple order in $E$ and with respect to this order $E$ is an ordered commutative idempotent semigroup.

6. If $e, f, g \in E, f \prec g \leqq e$ and $x \in R_{e}$, then $x^{-1} f x \prec x^{-1} g x$.

In fact, since $f \prec g$, we have $f g=f$ and so $x^{-1} f x=x^{-1} f g x=\left(x^{-1} f x\right)\left(x^{-1} g x\right)$. Hence $x^{-1} f x \leqq x^{-1} g x$. But, if $x^{-1} f x=x^{-1} g x$ were true, then $f=e f e=x x^{-1} f x x^{-1}$ $=x x^{-1} g x x^{-1}=e g e=g$, which is a contradiction. Hence $x^{-1} f x \prec x^{-1} g x$.

Now we prove that the ordered semigroup $E$ satisfies the condition (16') and suppose that $e, f, g \in E, x \in R_{e}, f \leqq e, g \leqq e$ and $f \leqq g$.

(i) The case when $f=g$. Clearly $x^{-1} f x=x^{-1} g x$.

(ii) The case when $f g=f \prec g$. Since $f \leqq g$ and $f g \neq g, g$ lies in the latter branch $B$ at $f g=f$. By $6^{\circ}$, we have $x^{-1} f x \prec x^{-1} g x$ and so $x^{-1} g x$ lies in some branch $B^{\prime}$ at $x^{-1} f x$. Now we have

$$
\begin{aligned}
& (f x)(f x)^{-1}=f x x^{-1} f=f e f=f, \quad(f x)^{-1}(f x)=x^{-1} f x, \\
& (g x)(g x)^{-1}=g x x^{-1} g=g e g=g \in B, \quad(g x)^{-1}(g x)=x^{-1} g x \in B^{\prime}, \\
& (f x)^{-1}(g x)=x^{-1} f g x \in E .
\end{aligned}
$$

Hence the branch $B$ is transferred to the branch $B^{\prime}$ by a translation. Therefore $B$ and $B^{\prime}$ lie in the same equivalence class and, since $B$ is the latter branch at $f, B^{\prime}$ is the latter branch at $x^{-1} f x$. Moreover $\left(x^{-1} f x\right)\left(x^{-1} g x\right)=x^{-1} f g x=x^{-1} f x \prec x^{-1} g x$. Hence $x^{-1} f x \leqq x^{-1} g x$.

(iii) The case when $f g=g \prec f$. In a similar way to (ii), we can prove that $x^{-1} f x$ $\leqq x^{-1} g x$.

(iv) The case when $f g \prec f$ and $f g \prec g$. We have $f g \prec f \leqq e$ and, by Lemma 1.1, $f \leqq f g \leqq g$. Since $f(f g)=f g \prec f$, we have, by (iii), $x^{-1} f x \leqq x^{-1} f g x$. Also since $(f g) g$ $=f g \prec g$, we have, by (ii), $x^{-1} f g x \leqq x^{-1} g x$. Hence $x^{-1} f x \leqq x^{-1} g x$. This completes the proof of Theorem 6.6.

THEOREM 6.7. Let $S$ be an inverse semigroup which contains no elements of finite order except idempotents. If the set $E$ of all idempotents of $S$ is an ordered commutative idempotent semigroup satisfying the condition (16') in Theorem 5.4 and if $S$ contains a subsemigroup $P$ such that $P \cap P^{-1}=E, P \cup P^{-1}=S$ and $x^{-1} P x \subseteq P$ for every $x \in S$, then we can define such an order in $S$ in one and only one way that $S$ is 
an ordered inverse semigroup, $P$ is the nonnegative part of the ordered inverse semigroup $S$ and, on the set $E$, the order in $S$ coincides with the given order in $E$.

Proof. First we prove the 'in one way' part. By Theorem 6.2, we can define an order in $S$ such that $S$ is a left ordered inverse semigroup, $P$ is the nonnegative part of the left ordered inverse semigroup $S$ and, on the set $E$, the order in $S$ coincides with the given order in $E$. It remains to prove that $S$ is an ordered inverse semigroup. Let $y$ be a positive element and let $x$ be an arbitrary element of $S$. Then $y \in P$ and so $x^{-1} y x \in x^{-1} P x \leqq P$. Hence $S$ satisfies the condition (151) in Theorem 5.6 and so, by Theorem 5.6, $S$ satisfies the condition (15') in Theorem 5.4. Moreover, by assumption, $S$ satisfies the condition $\left(16^{\prime}\right)$ in Theorem 5.4. Hence, by Theorem 5.4, $S$ is an ordered inverse semigroup. The 'in only one way' part of this theorem is included in the assertion of Theorem 6.2.

THEOREM 6.8. Let $S$ be an inverse semigroup and let $E$ be the set of all idempotents of $S$. Then $S$ is orderable if and only if $S$ satisfies the following three conditions:

(A) $S$ contains no elements of finite order except idempotents;

(B*) $S$ contains a subsemigroup $P$ such that $P \cap P^{-1}=E, P \cup P^{-1}=S$ and $x^{-1} P x \cong P$ for every $x \in S$;

$\left(C^{*}\right)$ the semilattice $E$ is a tree semilattice and, when a branch $B$ is connected with a branch $B^{\prime}$ and we choose branches $B=B_{1}, B_{2}, \ldots, B_{n}=B^{\prime}$ such that $B_{i}$ is transferred to $B_{i+1}$ by either a translation or a conversion for every $1 \leqq i \leqq n-1$, whether the number of conversions included in the transfers is even or odd is determined by $B$ and $B^{\prime}$, irrespective of the choice of branches $B_{i}(1 \leqq i \leqq n)$.

Proof. First we prove the 'only if' part. We suppose that $S$ is an ordered inverse semigroup. Then, by Theorem 6.3, $S$ satisfies the condition (A). We denote the nonnegative part of $S$ by $P$. As is shown in the proof of Theorem 6.3, $P$ is a subsemigroup of $S, P \cap P^{-1}=E$ and $P \cup P^{-1}=S$. Now we take $x \in S$ and $y \in P$ arbitrarily. If $y$ is idempotent, then $x^{-1} y x$ is idempotent and so $x^{-1} y x \in P$. By Theorem 5.4, $S$ satisfies the condition $\left(15^{\prime}\right)$ and so, by Theorem 5.6, $S$ satisfies the condition (151). Hence, if $y$ is positive, then $x^{-1} y x$ is nonnegative and so $x^{-1} y x \in P$. Therefore $x^{-1} P x \subseteq P$ and so $S$ satisfies the condition (B*). We showed that $S$ contains no elements of finite order except idempotents and, by Theorem 5.4, $S$ satisfies the condition $\left(16^{\prime}\right)$. Hence, by Theorem $6.6, S$ satisfies the condition (C*).

Next we prove the 'if' part. Suppose that $S$ is an inverse semigroup satisfying the conditions (A), ( $\left.\mathrm{B}^{*}\right)$ and $\left(\mathrm{C}^{*}\right)$. Then, by Theorem $6.6, E$ can be considered as an ordered commutative idempotent semigroup satisfying the condition (16') and so, by Theorem 6.7, $S$ can be considered as an ordered inverse semigroup.

THEOREM 6.9. Let $S$ be an inverse semigroup and let $E$ be the set of all idempotents of $S$. If $S$ satisfies the condition $\left(\mathrm{C}^{*}\right)$ in Theorem 6.8 , then $S$ satisfies the condition (C) in Theorem 6.3. 
Proof. Let $S$ be an inverse semigroup satisfying the condition $\left(C^{*}\right)$. By way of contradiction, we assume that, in the tree semilattice $E$, there exist at least three different branches $B, B^{\prime}$ and $B^{\prime \prime}$ at the same element $e \in E$. Then the transfers of $B$ to $B^{\prime}$ by $B=B_{1}, B^{\prime}=B_{2}$ contain one conversion, while the transfers of $B$ to $B^{\prime}$ by $B=B_{1}^{\prime}, B^{\prime \prime}=B_{2}^{\prime}, B^{\prime}=B_{3}^{\prime}$ contain two conversions, contradicting the condition $\left(\mathrm{C}^{*}\right)$.

7. Examples. The condition (B*) in Theorem 6.8 clearly implies the condition (B) in Theorem 6.3. Also, by Theorem 6.9, the condition $\left(\mathrm{C}^{*}\right)$ in Theorem 6.8 implies the condition (C) in Theorem 6.3. Examples in this section show that there are no other relations of implication among conditions (A), (B), (C), (B*) and $\left(C^{*}\right)$.

EXAMPLE 7.1. Let $S$ be a cyclic group of order three generated by an element $a$. We put $P=\{1, a\}$, where 1 is the identity of the group $S$. Then $P^{-1}=\left\{1, a^{2}\right\}$ and so $P \cap P^{-1}=\{1\}$ and $P \cup P^{-1}=S$. Since $S$ is a commutative group, we have $x^{-1} P x$ $=P$. Hence $S$ satisfies the condition $\left(\mathrm{B}^{*}\right)$ and also the condition (B). Moreover, since $S$ is a group, $S$ satisfies the conditions (C) and (C*) trivially. But clearly $S$ does not satisfy the condition (A).

EXAmple 7.2 ([6] and [7]). Let $S$ be the group generated by $\{b, u, v\}$ subject to the generating relations

$$
\begin{aligned}
{[[u, v], u] } & =[[u, v], v]=1, \quad b^{-1} u b=u^{-1}, \\
b^{-1} v b & =v^{-1}, \quad[u, v]=b^{-16},
\end{aligned}
$$

where, for $x, y \in S,[x, y]=x^{-1} y^{-1} x y$ and 1 is the identity of the group $S$. It was shown in [6] and [7] that $S$ satisfies the condition (A) but does not satisfy the condition (B). Since $S$ is a group, $S$ satisfies the conditions (C) and (C*) trivially.

EXAMPLE 7.3. Let $S$ be the semigroup consisting of four elements $\{0, a, b, c\}$ with the operation defined by

$$
\begin{aligned}
& \text { for } x, y \in S, \quad x y=x \quad \text { if } x=y \text {, } \\
& x y=0 \text { if } x \neq y \text {. }
\end{aligned}
$$

Then $S$ is a commutative idempotent semigroup and, in particular, is an inverse semigroup. Trivially $S$ satisfies the conditions (A), (B) and (B*). But the set of all idempotents of $S$ coincides with $S$ and, in the semilattice $S$, the branch number at 0 is three. Hence $S$ does not satisfy the condition (C).

EXAMPLE 7.4 [2]. Let $S$ be a system consisting of all pairs of integers with the operation

$$
(a, b)(c, d)=\left(a+c, b+(-1)^{a} d\right) .
$$

It was shown in [2] that $S$ is a group, $(0,0)$ is the identity of $S$ and the group inverse of $(a, b)$ is $\left(-a,-(-1)^{a} b\right)$. It is easily checked that $S$ satisfies the condition (A). Also $S$ satisfies (C) and (C*) trivially. Moreover $S$ satisfies the condition (B). In fact, it is easily shown that the set

$$
\{(a, b) \text {; either } a>0 \text { or } a=0, b \geqq 0\}
$$

satisfies the requirements for $P$ in the condition (B). But $S$ does not satisfy the 
condition $\left(\mathrm{B}^{*}\right)$. In fact, by way of contradiction, we assume that there is a subsemigroup $P$ of $S$ such that $P \cap P^{-1}=\{(0,0)\}, P \cup P^{-1}=S$ and $(a, b)^{-1} P(a, b)$ $\subseteq P$ for every $(a, b) \in S$. Since $(0,1) \in S=P \cup P^{-1}$, either $(0,1) \in P$ or $(0,-1)$ $=(0,1)^{-1} \in P$. But

$$
\begin{aligned}
(1,1)^{-1}(0,1)(1,1) & =(-1,1)(0,1)(1,1)=(0,-1), \\
(1,1)^{-1}(0,-1)(1,1) & =(-1,1)(0,-1)(1,1)=(0,1),
\end{aligned}
$$

and so, in both cases, we have $(0,1),(0,-1) \in P$. Hence $(0,1) \in P \cap P^{-1}=\{(0,0)\}$, which is a contradiction.

ExAmPLe 7.5. Let $M=\left\{a, b, c, d, e, f_{i}, g_{i} ; i=1,2,3, \ldots\right\}$. We put

$$
\pi(f)=\left(\begin{array}{l}
\cdots f_{2 n+1} \cdots f_{5} f_{3} f_{1} f_{2} f_{4} \cdots f_{2 n} \cdots \\
\cdots f_{2 n-1} \cdots f_{3} f_{1} f_{2} f_{4} f_{6} \cdots f_{2 n+2} \cdots
\end{array}\right)
$$

Clearly $\pi(f)$ generates an infinite cyclic group with respect to the operation of composition of transformations on the set $\left\{f_{i} ; i=1,2,3, \ldots\right\}$. We denote $(\pi(f))^{m}$ by $\pi^{m}(f)$ for every integer $m$. Simliarly

$$
\pi(g)=\left(\begin{array}{l}
\cdots g_{2 n+1} \cdots g_{5} g_{3} g_{1} g_{2} g_{4} \cdots g_{2 n} \cdots \\
\cdots g_{2 n-1} \cdots g_{3} g_{1} g_{2} g_{4} g_{6} \cdots g_{2 n+2} \cdots
\end{array}\right)
$$

generates an infinite cyclic group and it consists of elements $\pi^{m}(g)=(\pi(g))^{m}(m$, an integer). Let $S$ be the set consisting of the following partial one-to-one transformations on the set $M$ :

$$
\begin{aligned}
& x_{1}=\left(\begin{array}{lll}
a d & d \\
a & d
\end{array} \quad \pi^{0}(f) ; \quad \pi^{0}(g)\right), \quad x_{6}=\left(\begin{array}{ll}
b d & d \\
c & e
\end{array} \quad \pi^{1}(f) ; \quad \pi^{0}(g)\right), \\
& x_{2}=\left(\begin{array}{lll}
a & d \\
b & d
\end{array} \quad \pi^{-1}(f) ; \quad \pi^{1}(g)\right), \quad x_{7}=\left(\begin{array}{llll}
c & e \\
a & d
\end{array} \quad \pi^{0}(f) ; \quad \pi^{-1}(g)\right),
\end{aligned}
$$

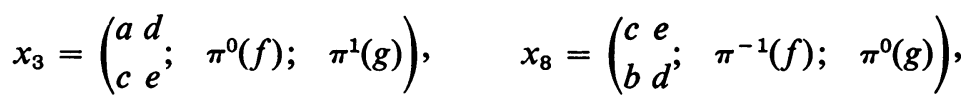

$$
\begin{aligned}
& x_{4}=\left(\begin{array}{lll}
b & d \\
a & b
\end{array} ; \quad \pi^{1}(f) ; \quad \pi^{-1}(g)\right), \quad x_{9}=\left(\begin{array}{llll}
c & e \\
c & e
\end{array} \quad \pi^{0}(f) ; \quad \pi^{0}(g)\right), \\
& x_{5}=\left(\begin{array}{lll}
b & d \\
b & d
\end{array} ; \pi^{0}(f) ; \quad \pi^{0}(g)\right)
\end{aligned}
$$

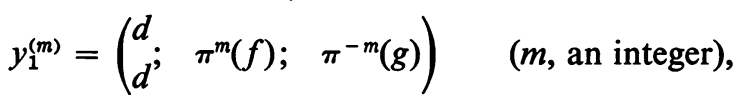

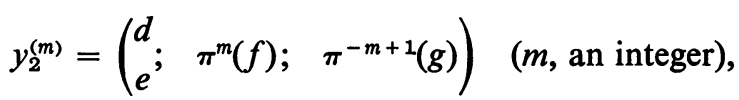

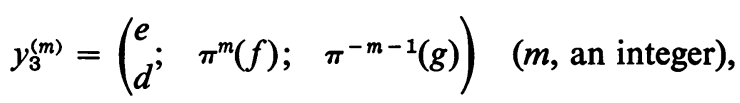

$$
\begin{aligned}
& y_{4}^{(m)}=\left(\begin{array}{lll}
e & \pi^{m}(f) ; & \pi^{-m}(g) \\
e^{\prime} &
\end{array} \quad(m, \text { an integer }),\right. \\
& z^{(m, n)}=\left(\pi^{m}(f) ; \pi^{n}(g)\right) \quad(m, n, \text { integers }) .
\end{aligned}
$$


It can be shown that $S$ forms an inverse semigroup with respect to the operation of composition of partial transformations. Also we can show that $x_{1}^{-1}=x_{1}, x_{2}^{-1}=x_{4}$, $x_{3}^{-1}=x_{7}, \quad x_{4}^{-1}=x_{2}, \quad x_{5}^{-1}=x_{5}, \quad x_{6}^{-1}=x_{8}, \quad x_{7}^{-1}=x_{3}, \quad x_{8}^{-1}=x_{6}, \quad x_{9}^{-1}=x_{9}, \quad\left(y_{1}^{(m)}\right)^{-1}$ $=y_{1}^{(-m)},\left(y_{2}^{(m)}\right)^{-1}=y_{3}^{(-m)},\left(y_{3}^{(m)}\right)^{-1}=y_{2}^{(-m)},\left(y_{4}^{(m)}\right)^{-1}=y_{4}^{(-m)},\left(z^{(m, n)}\right)^{-1}=z^{(-m,-n)}$. The set $E$ of idempotents of $S$ consists of $x_{1}, x_{5}, x_{9}, y_{1}^{(0)}, y_{4}^{(0)}, z^{(0,0)}$ and the semilattice $E$ has the following scheme:

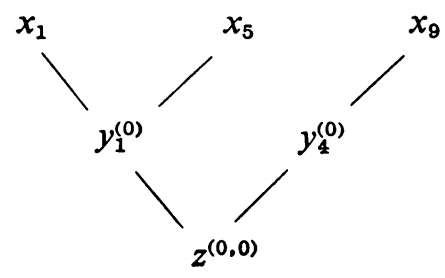

Thus $S$ satisfies the condition (C). Since $\pi^{m}(f)$ and $\pi^{m}(g)$ have infinite order for $m \neq 0$, it can be seen that $S$ satisfies the condition (A). Now we show that $S$ satisfies the condition (B*) and so also the condition (B). In fact, when we denote by $P$ the set consisting of all elements of $S$, which have the $\pi^{m}(f)$-component and the $\pi^{n}(g)$ component with either $m>0$ or $m=0, n \geqq 0$, it is easily verified that $P$ satisfies all the requirements for $P$ in the condition $\left(\mathrm{B}^{*}\right)$. Finally we show that $S$ does not satisfy the condition ( $\left.\mathrm{C}^{*}\right)$. In fact, we put $x=y_{2}^{(0)}, y=x_{3}, u=y_{2}^{(1)}, v=x_{6}$. Then we have

$$
\begin{array}{rlrl}
x x^{-1} & =y_{2}^{(0)} y_{3}^{(0)}=y_{1}^{(0)}, & x^{-1} x=y_{3}^{(0)} y_{2}^{(0)}=y_{4}^{(0)}, \\
y y^{-1}=x_{3} x_{7}=x_{1}, & y^{-1} y=x_{7} x_{3}=x_{9}, \\
x^{-1} y=y_{3}^{(0)} x_{3}=y_{4}^{(0)} \in E, & \\
u u^{-1}=y_{2}^{(1)} y_{3}^{(-1)}=y_{1}^{(0)}, & u^{-1} u=y_{3}^{(-1)} y_{2}^{(1)}=y_{4}^{(0)}, \\
v v^{-1}=x_{6} x_{8}=x_{5}, & v^{-1} v=x_{8} x_{6}=x_{9}, \\
u^{-1} v=y_{3}^{(-1)} x_{6}=y_{4}^{(0)} \in E . &
\end{array}
$$

Hence the branch $B_{1}$ at $y_{1}^{(0)}$ which contains $x_{1}$ is transferred to the branch $B_{3}$ at $y_{4}^{(0)}$ which contains $x_{9}$ by a translation. Moreover the branch $B_{2}$ at $y_{1}^{(0)}$ which contains $x_{5}$ is transferred to the branch $B_{3}$ by a translation. Thus, in one way, $B_{1}$ is transferred to $B_{3}$ directly by one translation and, in another way, by one conversion and one translation. This contradicts the condition $\left(C^{*}\right)$.

\section{REFERENCES}

1. A. H. Clifford and G. B. Preston, The algebraic theory of semigroups. Vol. I, Math. Surveys, no. 7, Amer. Math. Soc., Providence, R. I., 1961. MR 24 \#A2627.

2. P. Conrad, Right-ordered groups, Michigan Math. J. 6 (1959), 267-275. MR 21 \#5684.

3. T. Saitô, Ordered idempotent semigroups, J. Math. Soc. Japan 14 (1962), 150-169. MR 26 \#2533.

4. - - Regular elements in an ordered semigroup, Pacific J. Math. 13 (1963), 263-295. MR 27 \#2574. 
5. T. Saitô, Proper ordered inverse semigroups, Pacific J. Math. 15 (1965), 649-666. MR 33 \#204.

6. D. M. Smirnov, One-sided orders in groups with ascending central series, Algebra i Logika Sem. 6 (1967), no. 2, 77-88. (Russian) MR 35 \#5372.

7. J. Wiegold, Semigroup coverings of groups. II, Mat.-Fyz. Casopis Sloven. Akad. Vied 12 (1962), 217-223. MR 28 \#2168.

TOKYO GaKUgeI UNIVERSITY,

TOKYO, JAPAN 